\title{
THE IDEAL STRUCTURE OF CERTAIN NONSELFADJOINT OPERATOR ALGEBRAS
}

\author{
JUSTIN PETERS
}

\begin{abstract}
Let $(X, \phi)$ be a locally compact dynamical system, and $\mathbf{Z}^{+} \times_{\phi}$ $C_{0}(X)$ the norm-closed subalgebra of the crossed product $\mathbf{Z} \times{ }_{\phi} C_{0}(X)$ generated by the nonnegative powers of $\phi$ in case $\phi$ is a homeomorphism. If $\phi$ is just a continuous map, $\mathbf{Z}^{+} \times{ }_{\phi} C_{0}(X)$ can still be defined by a crossed product type construction. The ideal structure of these algebras is determined in case $\phi$ acts freely. A class of strictly transitive Banach modules is described, indicating that for the nonselfadjoint operator algebras considered here, not all irreducible representations are on Hilbert space. Finally in a special case, the family of all invariant maximal right ideals is given.
\end{abstract}

\section{Introduction.}

I.1. Nearly twenty years ago Effros and Hahn [5] investigated the primitive ideal structure of certain $C^{*}$-crossed products, and their results have been extensively generalized in recent years. However, the study of nonselfadjoint subalgebras of $C^{*}$-crossed products is much more recent, and yet the same questions regarding the interplay between topological dynamics and ideal theory can be asked in the nonselfadjoint case as well. For example, if $X$ is a locally compact metric space, $\phi$ a freely acting homeomorphism of $X$ and $\alpha$ the corresponding automorphism of $C_{0}(X), \alpha(f)=f \circ \phi$, then the primitive ideals of the $C^{*}$-crossed product $\mathbf{Z} \times_{\alpha}$ $C_{0}(X)$ correspond naturally with the space of "pseudo orbits", or orbit closures $\left\{\phi^{n}(x): n \in \mathbf{Z}\right\}(x \in X)$. Denote by $\mathbf{Z}^{+} \times_{\alpha} C_{0}(X)$ the closed subalgebra of $\mathbf{Z} \times{ }_{\alpha}$ $C_{0}(X)$ generated by the nonnegative powers of the automorphism. What can be said about the primitive ideals of this algebra? Unfortunately, it seems that knowing $\operatorname{Prim}\left(\mathbf{Z} \times{ }_{\alpha} C_{0}(X)\right)$ shed no light on this problem. Inherent in this problem is the fact that $\mathbf{Z}^{+} \times_{\alpha} C_{0}(X)$ is not a star-algebra. And although the norm in $\mathbf{Z}^{+} \times{ }_{\alpha} C_{0}(X)$ is defined by means of Hilbert space representations, it turns out that the irreducible representations are not necessarily realizable in Hilbert space.

While for (separable) $C^{*}$-algebras, the primitive and prime ideals coincide, for a Banach algebra that need not happen. Returning to the algebra $\mathbf{Z}^{+} \times_{\alpha} C_{0}(X)$, we obtain results similar to those of Effros-Hahn for $C^{*}$-crossed products, but which require some topological dynamical refinements. For $x \in X$, let $\mathcal{O}^{+}(x)$ be the forward orbit $\left\{\phi^{n}(x): n=0,1, \ldots\right\}$. For example, the prime ideals of $\mathbf{Z}^{+} \times_{\alpha} C_{0}(X)$ correspond to $\left\{O^{+} \overline{(x)}: x\right.$ is a recurrent point $\}$. (Theorem III.10; cf. II. 8, II. 9 for definitions.)

Received by the editors October 20, 1985 and, in revised form, November 11, 1986.

1980 Mathematics Subject Classification (1985 Revision). Primary 46L55; Secondary 46H20, $54 \mathrm{H} 20$.

Key words and phrases. Dynamical system, nonselfadjoint operator algebra, free action, Banach module. 
I.2. Notation. The framework of our results is actually somewhat more general than that alluded to above. Let $X$ be a locally compact metric space; instead of requiring a homeomorphism for the dynamics, we only require that $\phi: X \rightarrow X$ be a continuous and proper surjection. That gives rise to a continuous injective (star) endomorphism $\alpha$ of $C_{0}(X), \alpha(f)=f \circ \phi$. Let $U$ be an operator defined formally by the condition $f U=U f \circ \phi, f \in C_{0}(X)$, and denote by $K\left(\mathbf{Z}^{+}, C_{0}(X)\right)$ the associative algebra over the complex field generated by $U$ and $C_{0}(X)$. A typical element $F$ of this algebra will have the form $F=f_{0}+U f_{1}+\cdots+U^{n} f_{n}$ for some nonnegative integer $n$ and functions $f_{0}, f_{1}, \ldots, f_{n} \in C_{0}(X)$. Let $l_{2}^{+}$be the space of square summable sequences $\xi=\left(\xi_{n}\right)_{n=0}^{\infty}, \xi_{n} \in \mathbf{C},\|\xi\|_{2}=\left(\sum_{n=0}^{\infty}\left|\xi_{n}\right|^{2}\right)^{1 / 2}$, and, for each $x \in X$, let $\Pi_{x}$ denote the representation of $K\left(\mathbf{Z}^{+}, C_{0}(X)\right)$ in $B\left(l_{2}^{+}\right)$given on the generators by

$$
\Pi_{x}(f) \xi=\left(f(x) \xi_{0}, f \circ \phi(x) \xi_{1}, f \circ \phi^{2}(x) \xi_{2}, \ldots\right)
$$

and

$$
\Pi_{x}(U) \xi=\left(0, \xi_{0}, \xi_{1}, \xi_{2}, \ldots\right) .
$$

A norm can be defined on $K\left(\mathbf{Z}^{+}, C_{0}(X)\right)$ by $\|F\|=\sup \left\{\left\|\Pi_{x}(F)\right\|: x \in X\right\}$. The completion of $K\left(\mathbf{Z}^{+}, C_{0}(X)\right)$ with respect to this norm is called the semicrossed product of $C_{0}(X)$ with $\phi$, and is denoted by $\mathbf{Z}^{+} \times_{\phi} C_{0}(X)$.

To $F \in \mathbf{Z}^{+} \times{ }_{\phi} C_{0}(X)$ there corresponds, formally, a unique series $\sum_{n=0}^{\infty} U^{n} f_{n}$ $\left(f_{n} \in C_{0}(X)\right)$ (see III.3), called the Fourier series of $F$; the $f_{n}$ are called the Fourier coefficients. Although the partial sums of $\sum_{n=0}^{\infty} U^{n} f_{n}$ may not converge, it is Cesàro summable to $F[\mathbf{1 1}$, II.2, Remark]. For convenience we will often write $F=\sum_{n=0}^{\infty} U^{n} f_{n}$.

I.3. Contents. §II contains examples and notions from topological dynamics which will be used later. The ideal structure of $\mathbf{Z}^{+} \times_{\phi} C_{0}(X)$ is determined in $\S$ III for a free dynamical system $(X, \phi)$. $I \mathrm{IV}$ concerns the conjugate space of $\mathbf{Z}^{+} \times_{\phi} C_{0}(X)$. A partial classification of irreducible Banach modules of $\mathbf{Z}^{+} \times{ }_{\phi} C_{0}(X)$ is presented in $\S \mathrm{V}$, and some related questions are discussed.

\section{Topological dynamics.}

II.1. By a dynamical system $(X, \phi)$ we mean a locally compact metrizable space $X$ together with a continuous and proper surjection $\phi: X \rightarrow X$. (Recall that a map $\phi$ is proper if the inverse image $\phi^{-1}(K)$ is compact for every compact $K \subset X$.) Let $\alpha$ be in the injective endomorphism of $C_{0}(X), \alpha(f)=f \circ \phi$.

II.2. DEFINITIONS. If $(X, \phi),(Y, \psi)$ are dynamical systems, $(Y, \psi)$ is a factor of $(X, \phi)$ if there is a continuous and proper surjection $p: X \rightarrow Y$ with $\psi \circ p=p \circ \phi$. We also say that $(X, \phi)$ is an extension of $(Y, \psi)$. By a covering system for a dynamical system $(X, \phi)$ we mean an extension $(\tilde{X}, \tilde{\phi})$ such that $\tilde{\phi}$ is a homeomorphism and if $\left(X_{1}, \phi_{1}\right)$ is any other extension of $(X, \phi)$ with $\phi_{1}$ a homeomorphism, then $(\tilde{X}, \tilde{\phi})$ is a factor of $\left(X_{1}, \phi_{1}\right)$. (This terminology is nonstandard.)

Although the existence of covering systems for dynamical systems is not new, it is perhaps worth noting that it follows from a general principle concerning extendability of endomorphisms. By Proposition I.8 of [11], if $\alpha$ is an injective endomorphism of a $C^{*}$-algebra $\mathfrak{A}$, there is a $C^{*}$-algebra $B$ containing $\mathfrak{A}$ as a subalgebra, and an automorphism $\beta$ of $B$ such that $\alpha(x)=\beta(x), x \in \mathfrak{A}$. Furthermore it follows (from the proof) that $(B, \beta)$ can be chosen so that $\bigcup_{n>0} \beta^{-n}(\mathfrak{A})$ is dense in $B$ and hence 
$B$ is commutative if $\mathfrak{A}$ is. So if $\mathfrak{A}=C_{0}(X), B=C_{0}(\tilde{X}), \beta(\tilde{f})=\tilde{f} \circ \tilde{\phi}$ for some homeomorphism $\tilde{\phi}$ of $\tilde{X}\left(\tilde{f} \in C_{0}(\tilde{X})\right)$. If $j: \mathfrak{A} \rightarrow B$ is the inclusion, take $p$ to be the transpose restricted to the extreme points of the probability measures on $\tilde{X}$. It is not hard to verify that $(\tilde{X}, \tilde{\phi})$ is a factor of any extension $\left(X_{1}, \phi_{1}\right)$ of $(X, \phi)$ where $\phi_{1}$ is a homeomorphism. Thus $(\tilde{X}, \tilde{\phi})$ is a covering system of $(X, \phi)$.

Now we recall some standard terminology from dynamical systems. Let $(X, \phi)$ be a dynamical system and $x_{0} \in X$. The point $x_{0}$ is said to be recurrent if, for any neighborhood $V$ of $x_{0}$, there is an integer $n \geq 1$ with $\phi^{n}(x) \in V$. (Definition 1.1 of [6].) A subset $S \subset\{0,1,2, \ldots\}=\mathbf{Z}^{+}$is called syndetic if there is a finite set $\mathcal{F} \subset \mathbf{Z}^{+}$such that $S+\mathcal{F}=\mathbf{Z}^{+}$. $x_{0}$ is called almost periodic if for any neighborhood $V$ of $x_{0},\left\{n \in \mathbf{Z}^{+}: \phi^{n}\left(x_{0}\right) \in V\right\}$ is syndetic. (Some authors, e.g. [6], call this property uniform recurrence.) A subset $A \subset X$ is called a wandering set if the sets $A, \phi^{-1}(A), \phi^{-2}(A), \ldots$ are pairwise disjoint. A point $x_{0} \in X$ is a wandering point if $x_{0}$ has an open neighborhood which is a wandering set. If $Y \subset X$ is closed and $\phi(Y)=Y,\left(Y,\left.\phi\right|_{Y}\right)$ (or simply $\left.(Y, \phi)\right)$ will be referred to as a subsystem of $(X, \phi)$.

As these concepts will be fundamental to the next section, it is appropriate to give some examples here.

EXAMPLE II.2. Fix an orthogonal coordinate system and a unit length in the Euclidean plane $\mathbf{R}^{2}$. Let $X_{n}$ be the circle in $\mathbf{R}^{2}$ centered at $(0, n+1 / n)$ with radius $n$, and $\mathbf{R}=\{(x, y): y=0\}$. Set $X=\bigcup_{n=1}^{\infty} X_{n} \cup \mathbf{R}$, and give $X$ the topology induced from $\mathbf{R}^{2}$. Define a homeomorphism $\phi: X \rightarrow X$ as follows: for each $n, \phi$ maps $X_{n}$ onto itself by counterclockwise rotation through an arc of unit length; $\phi$ maps $\mathbf{R}$ to $\mathbf{R}$ by $\phi(x, 0)=(x+1,0)$. Since $\phi$ rotates $X_{n}$ through an irrational angle, the forward orbit $\left\{\phi^{k}\left(x_{0}\right): k=0,1, \ldots\right\}$ of any $x_{0} \in X_{n}$ is dense in $X_{n}$, and $x_{0}$ is almost periodic. Since every point $(x, 0) \in \mathbf{R}$ is a limit of points of $X_{n}$ $(n \uparrow \infty)$, the almost periodic points of $X$ are dense. Although the system $(X, \phi)$ has no wandering points, every point of the subsystem $(\mathbf{R}, \phi)$ is a wandering point of the subsystem $(\mathbf{R}, \phi)$.

II.3. Here we will construct a dynamical system $(X, \phi)$ with $X$ compact, $\phi$ noninvertible, such that the recurrent points of $X$ form a dense subset and such that no recurrent point, with the exception of a single fixed point, is almost periodic.

Let $\Lambda$ be a finite alphabet and $\phi$ be the backward shift on $\Lambda^{\mathbf{z}^{+}}$: thus, $\phi(\omega)=\omega^{\prime}$, where $\omega^{\prime}(n)=\omega(n+1)$. A word $\omega \in \Lambda^{\mathbf{z}^{+}}$is recurrent iff $\omega$ has the form

$$
\left[\left(a \omega^{(1)} a\right) \omega^{(2)}\left(a \omega^{(1)} a\right)\right] \omega^{(3)}\left[\left(a \omega^{(1)} a\right) \omega^{(2)}\left(a \omega^{(1)} a\right)\right] \cdots
$$

where $a \in \Lambda$ and $\omega^{(1)}, \omega^{(2)}, \omega^{(3)}, \ldots$ are words of finite length [6, Proposition 1.10]. In this example we take $\Lambda=\{0,1\}, a=1$, and $\omega^{(n)}$ to be the word consisting of a block of $n$ zeros. Let $\omega$ have the form (*), and set $X=0^{+}(\omega)^{-}$; that is, $X$ is the closure in $\Lambda^{\mathbf{Z}^{+}}$of the forward orbit $\mathcal{O}^{+}(\omega)=\left\{\omega, \phi(\omega), \phi^{2}(\omega), \ldots\right\}$, and $(X, \phi)$ is a subsystem of $\left(\Lambda^{\mathbf{z}^{+}}, \phi\right)$. Set $\omega_{0}=(0,0,0, \ldots)$. Then $\omega_{0} \in X$; indeed, let $l_{n}$ be the number of letters which precede the first letter of (the first occurrence of) the word $\omega^{(n)}$ in $\omega$. Clearly, $\phi^{l_{n}}(\omega) \rightarrow \omega_{0}$. Next we show that $\omega_{0}$ is the only almost periodic point of $X$. For suppose $\omega_{1} \neq \omega_{0}$ is another almost periodic point of $X$, and let $\eta$ be a word (of finite length) in $\omega_{1}$ such that not all the letters of $\eta$ are zero. Now the occurrences of $\eta$ in $\omega_{1}$ form a syndetic set $[6,1.22]$; let $d(\eta)$ be the maximum difference between successive terms of this set. Specifically, let $S \subset \mathbf{Z}^{+}$ be defined as follows: $n \in S$ iff $\omega_{1}(n)$ is a letter in (some occurrence) of $\eta$ in $\omega_{1}$. 
So if $S=\left\{n_{1}, n_{2}, \ldots\right\}$, set $d(\eta)=\max \left\{n_{k+1}-n_{k}, k=1,2, \ldots\right\}$ which is finite since $S$ is syndetic. If $l(\eta)$ is the length of $\eta$ (i.e., the number of letters in $\eta$ ), set $p=l(\eta)+d(\eta)$. Then any subword of $\omega_{1}$ of length at least $p$ must contain $\eta$. For any $n \geq 1$, write $\phi^{n}(\omega)=\left(\omega^{\prime}, \omega^{\prime \prime}\right)$, where either $\omega^{\prime}$ is the empty word or else $\omega^{\prime}=(0,0, \ldots, 0)$ consisting of all zeros, and $\omega^{\prime \prime}=(1,0, \ldots)$. We claim that $\phi^{n}(\omega)$ $(n \geq 1)$ cannot agree with $\omega_{1}$ in the first $2 p+l_{p}$ letters. If the length of $\omega^{\prime}$ is at least $p$, this is obvious. So suppose $\omega^{\prime}$ has length less than $p$. The number of letters which precede the first occurrence of a word $\omega^{(k)}$ in $\omega^{\prime \prime}$, for some $k \geq p$, is at most $l_{p}$. This fact is an immediate consequence of the form $(*)$ of $\omega$. Thus, the first $l_{p}+p$ letters of $\omega^{\prime \prime}$ contain a block of $p$ zeros, and hence $\phi^{n}(\omega)$ cannot agree with $\omega_{1}$ in the first $l_{p}+2 p$ letters. Thus there is no sequence $\left\{n_{k}\right\}$ such that $\phi^{n_{k}}(\omega) \rightarrow \omega_{1}$, so $\omega_{1} \notin X$.

As $\omega$ is recurrent and $0^{+}(\omega)$ is dense in $X$, the set of recurrent points is dense and there are no wandering points. In fact, the set of recurrent points of $(X, \phi)$ is residual [6, Theorem 1.27$]$.

II.4. EXAMPLE. This is an example of a dynamical system $\left(X_{1}, \phi_{1}\right)$ with $X_{1}$ compact, $\phi_{1}$ a homeomorphism, and such that the set of almost periodic points of $X_{1}$ is not dense.

Let $(X, \phi)$ be the dynamical system constructed in the previous example, and let $\left(X_{1}, \phi_{1}\right)$ be a covering system of $(X, \phi)$. If $x_{1} \in X_{1}$ is almost periodic and $p: X_{1} \rightarrow X$ is a continuous surjection with $\phi \circ p=p \circ \phi_{1}$ then $p\left(x_{1}\right)$ is an almost periodic point of $X$. Since the only periodic point of $X$ is the (fixed) point $\omega_{0}$, it follows that any almost periodic point of $X_{1}$ is contained in the inverse image $p^{-1}\left(\omega_{0}\right)$, and in particular such points are not dense in $X_{1}$.

II.5. Let $(X, \phi)$ be a dynamical system. In [11] a point $x_{0} \in X$ was called nearly recurrent if for every neighborhood $V$ of $x_{0}$ there is a point $x_{1} \in V$ such that $\left\{n: \phi^{n}\left(x_{1}\right) \in V\right\}$ has infinite cardinality. $x_{0}$ was called nearly periodic if, for every neighborhood $V$ of $x_{0}$, there is a point $x_{1} \in V$ such that $\left\{n: \phi^{n}\left(x_{1}\right) \in V\right\}$ is syndetic.

PROPOSITION. Let $(X, \phi)$ be a dynamical system. Then

(a) If the set of wandering points of $X$ is empty, then the set of nearly recurrent points of $X$ is the closure of the set of recurrent points;

(b) The set of nearly periodic points of $X$ is the closure of the set of almost periodic points.

PrOOF. (a) Since the set of nearly recurrent points is a closed invariant set whose complement is the set of wandering points, by hypothesis all points of $X$ are nearly recurrent. If $X$ is not compact, replace $(X, \phi)$ by $\left(X_{1}, \phi_{1}\right)$, where $X_{1}=X \cup\{\infty\}$ is the one point compactification of $X$, and $\phi_{1}(x)=\phi(x), x \in X, \phi_{1}(\infty)=\infty$. Since $\phi$ is onto, so is $\phi_{1}$. To see $\phi_{1}$ is continuous, let $x_{n} \rightarrow \infty$. If $\phi\left(x_{n}\right)$ did not approach $\infty$, there would be a compact set $K$, an integer $N$ and a subsequence $\left(x_{n_{j}}\right)$ such that $\phi\left(x_{n_{j}}\right) \in K, n_{j} \geq N$. But since $\phi$ is proper, $\phi^{-1}(K)$ is compact, and $x_{n_{j}} \in \phi^{-1}(K), n_{j} \geq N$, a contradiction. Now by [6, Theorem 1.27], the set of nonrecurrent points is residual.

(b) Let $x_{0} \in X$ be nearly periodic and $V$ be a compact neighborhood of $x_{0}$. By definition, there is a point $x_{1} \in V$ such that the set $S=\left\{n: \phi^{n}\left(x_{1}\right) \in V\right\}$ is 
syndetic. If $S=\left\{n_{k}\right\}_{k=1}^{\infty}$, let $d=\max \left\{n_{k+1}-n_{k}: k \geq 1\right\}<\infty$. Then $\mathcal{O}^{+}\left(X_{1}\right) \subset$ $\bigcup_{j=0}^{d} \phi^{-j}(V) \equiv W$. Indeed, if $m>0$, let $n>m$ such that $n-m \leq d$ and $n \in S$. Then

$$
\phi^{m}\left(x_{1}\right) \in \phi^{-(n-m)}\left(\phi^{n}\left(x_{1}\right)\right) \subset \phi^{-(n-m)}(V) \subset W .
$$

Since $W$ is compact, $O^{+} \overline{\left(x_{1}\right)} \subset W$. By a theorem of Auslander and Ellis applied to the compact dynamical system $(Y, \phi), Y=0^{+} \overline{\left(x_{1}\right)}, Y$ contains an almost periodic point, say $y_{0}\left[\mathbf{6}\right.$, Theorem 8.7]. Thus $y_{0} \in \phi^{-k}(V)$ for some $k, 0 \leq k \leq d$. Since $y_{0}$ is almost periodic, so is every point of $0^{+}\left(y_{0}\right)$, in particular $\phi^{k}\left(y_{0}\right)$. As $\phi^{k}\left(y_{0}\right) \in V$, and $V$ was an arbitrary compact neighborhood of $x_{0}$, it follows that the almost periodic points are dense in the nearly periodic points.

In the context of group actions, Gottschalk and Hedlund have used the term regionally recurrent for what we have called nearly recurrent $[\mathbf{7}, 7.17]$. Thus, if we were considering group actions instead of the semigroup $\mathbf{Z}^{+}$, part (a) of the proposition would follow from [7, Theorem 7.20].

II.6. There is a mistake in the proof of part (ii) of Theorem IV.1 of [11], and the "iff" in that statement should be replaced by "if". The question of necessary and sufficient conditions on the dynamical system for the semicrossed product to be semisimple is still open.

THEOREM. Let $(X, \phi)$ be a dynamical system, and $\mathfrak{A}=\mathbf{Z}^{+} \times_{\phi} C_{0}(X)$ the associated semicrossed product. Then

(i) $\mathfrak{A}$ is semiprime iff the recurrent points of $(X, \phi)$ are dense;

(ii) $\mathfrak{A}$ is semisimple if the almost periodic points of $(X, \phi)$ are dense;

(iii) $\mathfrak{A}$ is strongly semisimple iff the periodic points of $(X, \phi)$ are dense;

(iv) The normed algebra $K\left(\mathbf{Z}^{+}, C_{0}(X)\right)$ is semisimple iff the almost periodic points of $(X, \phi)$ are dense.

PrOOF OF (iv). Let $K$ be a compact neighborhood contained in the complement of the closure of the almost periodic points (equivalently, the complement of the nearly periodic points). The set $J$ of all $F \in K\left(\mathbf{Z}^{+}, C_{0}(X)\right)$ whose Fourier coefficients are all supported on $K$ is a left ideal in $K\left(\mathbf{Z}^{+}, C_{0}(X)\right)$. Since the radical contains every right or left nil ideal [12, 2.3.5], it follows that $J$ belongs to the radical, since the calculation on the top of p. 528 of [11] shows precisely that every $F \in J$ is nilpotent. Thus the radical of $K\left(\mathbf{Z}^{+}, C_{0}(X)\right)$ is nontrivial.

On the other hand, suppose every point of $X$ is nearly periodic, and let $R$ be the radical of $K\left(\mathbf{Z}^{+}, C_{0}(X)\right)$. If $K(X)$ denotes the continuous functions with compact support, then $R \neq(0)$ implies $R \cap K\left(\mathbf{Z}^{+}, K(X)\right) \neq(0)$, since if $F \in R$ then $F u \in R$ and the Fourier coefficients of $F u$ have compact support if $u \in K(X)$; also if $F \neq 0, F u$ will be nonzero for some $u \in K(X)$. Lemma III.2 shows that if $F \in R \cap K\left(\mathbf{Z}^{+}, K(X)\right), F=\sum_{k=0}^{n} U^{k} f_{k}$, then $U^{k} f_{k} \in R \cap K\left(\mathbf{Z}^{+}, K(X)\right)$ for all $k=0,1, \ldots, n$. But then the calculation at the bottom of p. 528 of $[11]$ shows that if $F \neq 0, F$ cannot belong to any topologically nilpotent ideal. It follows from [12, Theorem 2.3.4] that the radical is trivial.

II.7. Next we give an example of a dynamical system $(X, \phi)$ such that the algebra $K\left(\mathbf{Z}^{+}, C_{0}(X)\right)$ is not semisimple. Let $(X, \phi),\left(X_{1}, \phi_{1}\right)$ be the dynamical systems constructed in II.3, II.4 resp. In both cases, we saw that the almost periodic points were not dense. Thus, by Theorem II.6(iv), it follows that $K(X, C(X))$ and $K\left(X_{1}, C\left(X_{1}\right)\right)$ are not semisimple algebras. Whether or not $\mathbf{Z}^{+} \times_{\phi} C(X)$ and $\mathbf{Z}^{+} \times_{\phi_{1}} C\left(X_{1}\right)$ are semisimple is not clear. 
II.8. A dynamical system $(X, \phi)$ is said to be topologically transitive if there exists a point $x_{0} \in X$ such that $O^{+} \overline{\left(x_{0}\right)}=X$. The following proposition reduces to $[\mathbf{1 4}$, Theorem 5.4] when $X$ is compact and $\phi$ a homeomorphism. The proof is omitted.

PROPOSITION. The following are equivalent:

(i) $(X, \phi)$ is topologically transitive;

(ii) If $E \subset X$ is closed, $\phi(E) \subset E, E \neq X$, then $E$ is nowhere dense;

(iii) If $U \subset X$ is open, nonempty, $\phi^{-1}(U) \subset U$, then $U$ is dense.

(iv) If $U, V$ are nonempty open sets in $X$, there exists $n \in \mathbf{Z}^{+}$such that $\phi^{-n}(U) \cap$ $V \neq \varnothing$

(v) $\{x: 0+\overline{(x)} \neq X\}$ is a set of first category.

II.9. A dynamical system $(X, \phi)$ is minimal if no proper closed subset is invariant under $\phi$; equivalently, every orbit $\mathcal{O}^{+}(x)$ is dense in $X(x \in X)$. We do not know any examples where $X$ is noncompact. If $(X, \phi)$ is a minimal dynamical system with $X$ compact, we will say $(X, \phi)$ is compact minimal, and in that case every $x \in X$ is almost periodic. For any dynamical system $(X, \phi)$ with $X$ compact, the set of almost periodic points is nonempty. If $x$ is an almost periodic point of a dynamical system $(X, \phi)$, its forward orbit closure $\mathcal{O}^{+} \overline{(x)}$ is a minimal invariant closed subset of $X[6,1.4]$. The following simple fact will be used later.

LEMMA. The dynamical system $(X, \phi)$ is compact minimal iff for every open set $O \subset X$, there exists a positive integer $N$ such that $\bigcup_{k=0}^{N} \phi^{-k}(0)=X$.

Recall that the support of a nonnegative Borel measure on a separable metric space is the complement of the union of all open sets of measure zero. A dynamical system $(X, \phi)$ is uniquely ergodic if there is a unique invariant probability measure on $X$. The support of an invariant measure is an invariant subset, and if $(X, \phi)$ is uniquely ergodic and $Y \subset X$ is the support of the invariant measure, then $(Y, \phi)$ is minimal. In particular, if $(X, \phi)$ is minimal and uniquely ergodic with invariant measure $\mu$, then $\mu(0)>0$ for every open set $0 \subset X$.

If $(X, \phi)$ is a dynamical system such that the family $\left\{\phi^{n}: n=1,2, \ldots\right\}$ is equicontinuous, then it is uniquely ergodic [4, Theorem 3.1]. But the converse is false, even if $(X, \phi)$ is compact minimal (e.g., let $X=T^{2}$, the two-torus, and let $\phi$ be the "skew product", $\phi(z, w)=\left(e^{2 \pi i \alpha} z, z w\right), \alpha$ a fixed irrational [3, p. 52]). In general, a compact minimal dynamical system need not be uniquely ergodic.

\section{Ideal structure.}

III.1. Let $(X, \phi)$ be a dynamical system, and let $B_{b}(X)$ denote the bounded Borel functions on $X$. If $F$ is an element of the algebra $K\left(\mathbf{Z}^{+}, B_{b}(X)\right)$ generated by the operator $U$ and $B_{b}(X)$, say $F=\sum_{n=0}^{N} U^{n} f_{n}\left(f_{n} \in B_{b}(X)\right)$, the representations $\Pi_{x}$ of $\S$ I can be defined on $K\left(\mathbf{Z}^{+}, B_{b}(X)\right)$ by the same formulas given earlier, and we set $\|F\|=\sup _{x \in X}\left\|\Pi_{x}(F)\right\|$. The completion $\mathbf{Z}^{+} \times_{\phi} B_{b}(X)$ of $K\left(\mathbf{Z}^{+}, B_{b}(S)\right)$ with respect to this norm is an operator algebra containing $\mathbf{Z}^{+} \times_{\phi} C_{0}(X)$ as a (norm closed) subalgebra.

LEMMA. Let $h_{i}, k_{i} \in B_{b}(X), 1 \leq i \leq m$, and assume

$$
\sum_{i=1}^{m}\left|h_{i}(x)\right|^{2} \leq 1, \quad \sum_{i=1}^{m}\left|k_{i}(x)\right|^{2} \leq 1 \quad \text { for all } x \in X .
$$


If $F_{i} \in \mathbf{Z}^{+} \times_{\phi} B_{b}(X), 1 \leq i \leq m$, then $\left\|\sum_{i=1}^{m} h_{i} F_{i} k_{i}\right\| \leq \sup _{i}\left\{\left\|F_{i}\right\|\right\}$.

ProOF. Let $G=\sum_{i=1}^{m} h_{i} F_{i} k_{i}$, and let $\xi, \eta \in l_{2}^{+}, \xi=\left(\xi_{n}\right)_{n=0}^{\infty}, \eta=\left(\eta_{n}\right)_{n=0}^{\infty}$, $\|\xi\|=\|\eta\|=1$. Then

$$
\begin{aligned}
\left(\Pi_{x}(G) \xi, \eta\right) & =\sum_{i=1}^{m}\left(\Pi_{x}\left(h_{i} F_{i} k_{i}\right) \xi, \eta\right) \\
& =\sum_{i=1}^{m}\left(\Pi_{x}\left(F_{i}\right) \Pi_{x}\left(k_{i}\right) \xi, \Pi_{x}\left(\overline{h_{i}}\right) \eta\right) \\
& =\left(\left[\begin{array}{lll}
\Pi_{x}\left(F_{i}\right) & & \\
& \ddots & \\
& & \Pi_{x}\left(F_{m}\right)
\end{array}\right]\left[\begin{array}{l}
\xi^{(1)} \\
\xi^{(m)}
\end{array}\right],\left[\begin{array}{l}
\eta^{(1)} \\
\eta^{(m)}
\end{array}\right]\right)
\end{aligned}
$$

where $\xi^{(i)}=\Pi_{x}\left(k_{i}\right) \xi, \eta^{(i)}=\Pi_{x}\left(\overline{h_{i}}\right) \eta$. Now $\xi^{(i)}=\left(k_{i}(x) \xi_{0}, k_{i} \circ \phi(x) \xi_{1}, k_{i}\right.$ 。 $\left.\phi^{2}(x) \xi_{2}, \ldots\right)$ and so $\left\|\xi^{(i)}\right\|^{2}=\sum_{n=0}^{\infty}\left|k_{i} \circ \phi^{n}(x)\right|^{2}\left|\xi_{n}\right|^{2}$; hence,

$$
\begin{aligned}
\sum_{i=1}^{m}\left\|\xi^{(i)}\right\|^{2} & =\sum_{i=1}^{m} \sum_{n=0}^{\infty}\left|k_{i} \circ \phi^{n}(x)\right|^{2}\left|\xi_{n}\right|^{2} \\
& =\sum_{n=0}^{\infty}\left(\sum_{i=1}^{m}\left|k_{i} \circ \phi^{n}(x)\right|^{2}\right)\left|\xi_{n}\right|^{2} \\
& \leq \sum_{n=0}^{\infty}\left|\xi_{n}\right|^{2} \leq 1 .
\end{aligned}
$$

Similarly, $\sum_{i=1}^{m}\left\|\eta^{(i)}\right\|^{2} \leq 1$. Thus the vectors $\left[\xi^{(1)}, \ldots, \xi^{(m)}\right],\left[\eta^{(1)}, \ldots, \eta^{(m)}\right]$ both have norm at most one. It follows that

$$
\left\|\Pi_{x}(G)\right\| \leq\left\|\left[\begin{array}{lll}
\Pi_{x}\left(F_{1}\right) & & \\
& \ddots & \\
& & \Pi_{x}\left(F_{m}\right)
\end{array}\right]\right\| \leq \sup _{i}\left\{\left\|\Pi_{x}\left(F_{i}\right)\right\|\right\} .
$$

III.2. Let $(X, \phi)$ be a dynamical system which has no periodic points; i.e., $\phi^{n}(x) \neq$ $x, n=1,2, \ldots$, all $x \in X$. This is also expressed by saying that $\phi$ acts freely. Let $K(X)$ denote the continuous functions with compact support on $X$.

LEMMA. Given $F \in K\left(\mathbf{Z}^{+}, K(X)\right)$ and $N \in \mathbf{Z}^{+}$there exist $m \in \mathbf{Z}^{+}$and $h_{i} \in$ $K(X), 1 \leq i \leq m$, such that, if $F=\sum_{k=0}^{n} U^{k} f_{k}$, then $\sum_{i=1}^{m} h_{i} F h_{i} \circ \phi^{N}=U^{N} f_{N}$. $\left(f_{N}\right.$ is understood to be zero if $N>n$.)

PROOF. Let $K=\bigcup_{k=0}^{n} \operatorname{supp}\left(f_{k}\right)$. Since $\phi$ acts freely, given $x \in \phi^{N}(K)$ there exists a compact neighborhood $V_{x}$ of $x$ such that, if $n^{\prime}=\max \{n, N\}, V_{x}, \phi^{-1}\left(V_{x}\right), \ldots$, $\phi^{-n^{\prime}}\left(V_{x}\right)$ are pairwise disjoint compact neighborhoods. As $\left\{\operatorname{int}\left(V_{x}\right): x \in \phi^{N}(K)\right\}$ is an open cover of $\phi^{N}(K)$, we can extract a finite subcover, $V_{1}, \ldots, V_{m}$. Let $\left\{h_{1}, \ldots, h_{m}\right\} \subset C_{0}(X)$ satisfy $0 \leq h_{i} \leq 1$, supp $h_{i} \subset V_{i}, 1 \leq i \leq m$, and 
$\sum_{i=1}^{m} h_{i}(x)^{2}=1, x \in \phi^{N}(K)$. (Choose a partition of unity $\left\{h_{i}^{\prime}\right\}$ for $\phi^{N}(K)$ subordinate to $\left\{V_{i}\right\}$, and set $h_{i}=\sqrt{h_{i}^{\prime}}$.) Compute

$$
\begin{aligned}
\sum_{i=1}^{m} h_{i} F h_{i} \circ \phi^{N} & =\sum_{i=1}^{m} h_{i}\left(\sum_{k=0}^{n} U^{k} f_{k}\right) h_{i} \circ \phi^{N} \\
& =\sum_{k=0}^{n} \sum_{i=1}^{m} h_{i} U^{k} f_{k} h_{i} \circ \phi^{N} \\
& =\sum_{k=0}^{n} \sum_{i=1}^{m} U^{k} h_{i} \circ \phi^{k} h_{i} \circ \phi^{N} f_{k} .
\end{aligned}
$$

Now $h_{i} \circ \phi^{k}$ is supported on $\phi^{-k}\left(V_{i}\right)$, which is disjoint from $\phi^{-N}\left(V_{i}\right)$ if $k \neq N$. Thus

$$
\sum_{i=1}^{m} h_{i} F h_{i} \circ \phi^{N}=\sum_{i=1}^{m} U^{N}\left(h_{i} \circ \phi^{N}\right)^{2} f_{N}=U^{N} f_{N} .
$$

III.3. LemMA. Let $(X, \phi)$ be a dynamical system. For each $n \geq 0$ there is a linear projection $P_{n}: \mathbf{Z}^{+} \times_{\phi} C_{0}(X) \rightarrow C_{0}(X)$ with the following properties:

(i) $P_{n}(f F)=f \circ \phi^{n} P_{n}(F)$, and $P_{n}(F f)=P_{n}(F) f, F \in \mathbf{Z}^{+} \times_{\phi} C_{0}(X), f \in$ $C_{0}(X)$

(ii) $\left\|P_{n}(F)\right\| \leq\|F\|, F \in \mathbf{Z}^{+} \times_{\phi} C_{0}(X)$;

(iii) If $F=\sum_{n=0}^{N} U^{n} f_{n}$, then $P_{n}(F)=f_{n}$; in general, for $F \in \mathbf{Z}^{+} \times_{\phi} C_{0}(X)$,

$$
F-\sum_{n=0}^{N} U^{n} P_{n}(F) \in\left(U^{N+1}\left(\mathbf{Z}^{+} \times_{\phi} C_{0}(X)\right) .\right.
$$

Also, the statements are valid if " $B_{b}(X)$ " is substituted for " $C_{0}(X)$ ".

ProOF. The automorphism $\tau_{t}$, defined on $K\left(\mathbf{Z}^{+}, C_{0}(X)\right)$ by $\tau_{t}\left(\sum_{n=0}^{N} U^{n} f_{n}\right)=$ $\sum_{n=0}^{N} U^{n} e^{i n t} f_{n}$, is isometric and hence extends to an automorphism of $\mathbf{Z}^{+} \times_{\phi}$ $C_{0}(X)$. Define $P_{n}(F)$ by $U^{n} P_{n}(f)=(1 / 2 \pi) \int_{0}^{2 \pi} \tau_{t}(F) e^{-i n t} d t$. It is shown in [10 and 11] that $P_{n}$ has the required properties.

III.4. THEOREM. Let $(X, \phi)$ be a dynamical system. Then $\phi$ acts freely if and only if every closed, two sided ideal $\mathrm{J} \subset \mathbf{Z}^{+} \times_{\phi} C_{0}(X)$ has the property

$$
F \in J \text { implies } U^{n} P_{n}(F) \in J, \quad n=0,1,2, \ldots
$$

Proof. Assume $\phi$ acts freely, and let $J \subset \mathbf{Z}^{+} \times_{\phi} C_{0}(X)$ be a closed, two sided ideal. Let $F \in J$ and $u \in C_{0}(X)$ with compact support, $0 \leq u \leq 1$. Now $F \in J$ implies $F u \in J$, and if the conclusion holds for $F u$, viz., $P_{n}(F u)=P_{n}(F) u \in J$, $n=0,1, \ldots$, then $P_{n}(F) \in J$. For let $u$ run through an approximate identity for $C_{0}(X)$, and use the fact that $J$ is closed.

Thus we assume $F \in J$ and there is a compact set $K$ such that $f_{n}=P_{n}(F)$ is supported on $K, n=0,1, \ldots$ Write $F=F^{\prime}+F^{\prime \prime}, F^{\prime}=\sum_{k=0}^{N} U^{k} f_{k}, F^{\prime \prime}=F-F^{\prime}$ $\left(N \in \mathbf{Z}^{+}\right.$is arbitrary but fixed). By Lemma III.2 there exist functions $h_{i} \in K(X)$, $1 \leq i \leq m$, such that $\sum_{i=1}^{m} h_{i} F^{\prime} h_{i} \circ \phi^{N}=U^{N} f_{N}$. Thus,

$$
\sum_{i=1}^{m} h_{i} F h_{i} \circ \phi^{N}=U^{N} f_{N}+F_{N}, \quad F_{N}=\sum_{i=1}^{m} h_{i} F^{\prime \prime} h_{i} \circ \phi^{N} \in U^{N+1}\left(\mathbf{Z}^{+} \times_{\phi} C_{0}(X)\right) \text {, }
$$


and $P_{n}\left(F_{N}\right)$ is supported on $K$, for all $n$. Given $\varepsilon>0$ there exists $G \in K\left(\mathbf{Z}^{+}, C_{0}(X)\right)$, $G=\sum_{k=N+1}^{M} U^{k} g_{k}$, supp $g_{k} \subset K$ all $k$, such that $\left\|F_{N}-G\right\|<\varepsilon$. Apply Lemma III.2 again, this time to $U^{N} f_{N}+G$. Thus there exist functions $k_{i} \in C_{0}(X), 0 \leq k_{1} \leq 1$, $1 \leq i \leq p, \sum_{i=1}^{p} k_{i}(x)^{2}=1$ for all $x \in K$. Now $U^{N} f_{N}+F_{N} \in J$, as is

$$
\sum_{i=1}^{p} k_{i}\left(U^{N} f_{N}+F_{N}\right) k_{i} \circ \phi^{N}=U^{N} f_{N}+\sum_{i=1}^{p} k_{i} F_{N} k_{i} \circ \phi^{N} .
$$

Estimate

$$
\begin{aligned}
\left\|\sum_{i=1}^{p} k_{i} F_{N} k_{i} \circ \phi^{N}\right\| & =\left\|\sum_{i=1}^{p} k_{i}\left(F_{N}-G\right) k_{i} \circ \phi^{N}+\sum_{i=1}^{p} k_{i} G k_{i} \circ \phi^{N}\right\| \\
& \leq\left\|\sum_{i=1}^{p} k_{i}\left(F_{N}-G\right) k_{i} \circ \phi^{N}\right\|+\left\|\sum_{i=1}^{p} k_{i} G k_{i} \circ \phi^{N}\right\| \\
& \leq\left\|F_{N}-G\right\|<\varepsilon
\end{aligned}
$$

by Lemma III.1 and the fact $\sum_{i=1}^{p} k_{i} G k_{i} \circ \phi^{N}=0$. Since $J$ is closed, we conclude $U^{N} f_{N} \in J$.

For the converse, suppose $\phi$ does not act freely. In other words, there is a point $x \in X$ and a positive integer $p$ such that $\phi^{p}(x)=x$. Then, as discussed in [11, III], there are representations of $\mathbf{Z}^{+} \times_{\phi} C_{0}(X)$ as matrix algebras of holomorphic functions. The kernels of these representations do not have the property that $F \in J$ implies $U^{n} P_{n}(F) \in J$ for all $n$.

III.5. Suppose $(X, \phi)$ is a dynamical system where $\phi$ acts freely, and let $J \subset$ $\mathbf{Z}^{+} \times_{\phi} C_{0}(X)$ be a closed, two sided ideal. For $f \in C_{0}(X)$, let $Z(f)$ be the zero set of $f,\{x: f(x)=0\}$. Set

$$
X_{n}=\bigcap\left\{Z\left(P_{n}(F)\right): F \in J\right\}
$$

and

$$
J^{\prime}=\left\{F \in \mathbf{Z}^{+} \times_{\phi} C_{0}(X): Z\left(P_{n}(F)\right) \supseteq X_{n}, n=0,1, \ldots\right\} .
$$

COROLlary. $J=J^{\prime}$.

Proof. Clearly, $J \subseteq J^{\prime}$. On the other hand, observe that $F \in J$ iff $U^{n} P_{n}(F) \in$ $J, n=0,1, \ldots$ One direction is Theorem III.4. Conversely, if $U^{n} P_{n}(F) \in J$, then so are the $n$th arithmetic means of the sequence $\left\{U^{n} P_{n}(F)\right\}_{n=0}^{\infty}$, hence so is $F$ [11, IV.2, Remark]. Let $J_{n}=\left\{f \in C_{0}(X): U^{n} f \in J\right\}$. $J_{n}$ is a closed ideal in $C_{0}(X)$, hence there is a closed set $X_{n}^{\prime} \subseteq X$ such that $J_{n}=\left\{f \in C_{0}(X): f \mid X_{n}^{\prime}=0\right\}$. Furthermore,

$$
\begin{aligned}
X_{n}^{\prime} & =\bigcap\left\{\mathbf{Z}(f): U^{n} f \in J\right\} \\
& =\bigcap\left\{Z\left(P_{n}(F)\right): F \in J\right\}=X_{n} .
\end{aligned}
$$

Let $F \in J^{\prime}$, so $P_{n}(F) \mid X_{n}=0$, whence $U^{n} P_{n}(F) \in J, n=0,1, \ldots$, and finally $F \in J$. Thus $J=J^{\prime}$.

Notation. Let $\left(X_{n}\right)_{n=0}^{\infty}$ be the sequence of closed subsets of $X$ associated with the ideal $J$ as above; write this as $J \sim\left(X_{n}\right)_{n=0}^{\infty}$. Of course, not every sequence of closed subsets of $X$ is associated with an ideal $J \subset \mathbf{Z}^{+} \times_{\phi} C_{0}(X)$. Let $J \sim\left(X_{n}\right)_{n=0}^{\infty}$, and $U^{n} f \in J$; then both $U^{n+1} f, U^{n} f U=U^{n+1} f \circ \phi \in J$, so both $f, f \circ \phi$ must vanish 
on $X_{n+1}$. If $f$ is chosen so that $Z(f)=X_{n}$ for any $x \in X_{n+1}, f(x)=f \circ \phi(x)=0$ implies that both $x, \phi(x) \in X_{n}$. Thus

$$
X_{n+1} \cup \phi\left(X_{n+1}\right) \subset X_{n}
$$

Conversely, if $\left(X_{n}\right)_{n=0}^{\infty}$ is any sequence of closed subsets of $X$ satisfying $(*)$, then the subspace $J \sim\left(X_{n}\right)_{n=0}^{\infty}, J=\left\{F \in \mathbf{Z}^{+} \times_{\phi} C_{0}(X): P_{n}(F) \mid X_{n}=0\right\}$ is closed under left and right multiplication by $U$ and by functions $f \in C_{0}(X)$, hence is a closed, two sided ideal. This proves

THEOREM. Let $(X, \phi)$ be a dynamical system with $\phi$ acting freely. Then there is a one-to-one correspondence between closed, two sided ideals $\mathrm{J} \subset \mathrm{Z}^{+} \times_{\phi} C_{0}(X)$ and sequences $\left(X_{n}\right)_{n=0}^{\infty}$ of closed subsets of $X$ satisfying $(*)$.

III.6. Even if $\phi$ does not act freely on $X$, still, corresponding to every sequence $\left(X_{n}\right)_{n=0}^{\infty}$ of closed subsets of $X$ satisfying $(*)$ of III.5 there is a closed ideal $J$ of $\mathbf{Z}^{+} \times_{\phi} C_{0}(X)$. Recall that a (two sided) ideal $J$ is prime if, whenever $J_{1}, J_{2}$ are two sided ideals such that $J_{1} J_{2} \subset J$, either $J_{1} \subset J$ or $J_{2} \subset J$.

LEMMA. Let $J \subset \mathbf{Z}^{+} \times_{\phi} C_{0}(X)$ be a prime ideal, $J \sim\left(X_{n}\right)_{n=0}^{\infty}$. Then either $X_{n}=Z$ for some closed, invariant subset $Z \subset X, n=0,1, \ldots$, or $X_{0}$ consists of a single point and $X_{n}=\varnothing, n \geq 1$.

ProOF. Set $Y_{n}=X_{n+1}, n=0,1, \ldots$ As $\left(X_{n}\right)_{n=0}^{\infty}$ satisfies (*) of III.5, so does $\left(Y_{n}\right)_{n=0}^{\infty}$; let $J_{1}$ be the corresponding ideal. If $U^{n} f \in J_{1}$, that is, $f\left|Y_{n}=f\right| X_{n+1}=$ 0 , then $U\left(U^{n} f\right)=U^{n+1} f \in J$. Consequently, $J_{2} J_{1} \subset J$, where $J_{2}$ is the ideal generated by $U$; viz., $J_{2}=U\left(\mathbf{Z}^{+} \times_{\phi} C_{0}(X)\right)$. As $J$ is prime, either $J_{1} \subset J$ or $J_{2} \subset J$. Suppose now that the sequence $\left(X_{n}\right)_{n=0}^{\infty}$ is not constant; so for some $N$, $X_{N+1} \subset X_{N}$ but $X_{N+1} \neq X_{N}$. Let $f \in C_{0}(X)$ be such that $f\left|X_{N+1}=0, f\right| X_{N}$ is not identically zero. In that case, $U^{N} f \in J_{1}$, but $U^{N} f \notin J_{1}$ is not contained in $J$. Hence $J_{2} \subset J$. The quotient map $\mathbf{Z}^{+} \times_{\phi} C_{0}(X) \rightarrow \mathbf{Z}^{+} \times_{\phi} C_{0}(X) / J_{2} \cong C_{0}(X)$ maps $\mathbf{Z}^{+} \times_{\phi} C_{0}(X)$ onto $C_{0}(X)$, and as $J$ is a prime ideal in $\mathbf{Z}^{+} \times_{\phi} C_{0}(X)$ which contains $J_{2}$, its image is a prime ideal in $C_{0}(X)$. But the prime ideals in $C_{0}(X)$ are exactly the maximal ideals (of codimension one), so $X_{0}$ consists of a single point, and $X_{n}=\varnothing, n \geq 1$.

If the sequence $\left(X_{n}\right)_{n=0}^{\infty}$ is constant, so $X_{n}=Z$ for all $n$, then $Z$ satisfies $Z \cup \phi(Z) \subset Z$, so $\phi(Z) \subset Z$ and $Z$ is a closed, invariant subset.

Notation. If an ideal $J \subset \mathbf{Z}^{+} \times_{\phi} C_{0}(X)$ is associated with a sequence $\left(X_{n}\right)_{n=0}^{\infty}$, and $X_{n}=Z$ for all $n$, we will write $J \sim(Z)$.

III.7. Proposition. Let $(X, \phi)$ be a dynamical system, $J \subset \mathbf{Z}^{+} \times_{\phi} C_{0}(X) a$ prime ideal such that $J \sim(Z), Z \subset X$ a closed, invariant subset. Then $(Z, \phi)$ is transitive; that is, there exists $x_{0} \in Z$ such that $Z=0^{+} \overline{\left(x_{0}\right)}$.

PROOF. First we claim that the recurrent points are dense in $Z$, or equivalently (by Proposition II.5) that every point of $Z$ is nearly recurrent. Let $X_{n r}$ be the set of nearly recurrent points of $X$. If $X \backslash X_{n r}$ intersects $Z$, let $f \in C_{0}(X)$ be such that $f\left|X_{n r}=0, f\right| Z$ not identically zero. By $[11$, IV.4] $U f$ belongs to the prime radical of $\mathbf{Z}^{+} \times{ }_{\phi} C_{0}(X)$, but $U f \notin J$. This contradiction establishes the claim.

Let $Z^{(1)} \subset Z$ be a closed, invariant subset with nonempty interior (with respect to $Z$ ). Then $Z^{(2)}=\left(Z \backslash Z^{(1)}\right)^{-}$is also invariant: for the recurrent points are dense 
in the open set $Z \backslash Z^{(1)}$, hence are dense in $Z^{(2)}$ if $Z^{(2)}$ is nonempty. Suppose $x \in Z \backslash Z^{(1)}$ is recurrent; if $\phi(x) \in Z^{(1)}$ then by invariance of $Z^{(1)}, \phi^{n}(x) \in Z^{(1)}$ for all $n \geq 1$. By recurrence there is a sequence $\left\{n_{k}\right\}$ such that $\phi^{n_{k}}(x) \rightarrow X$, so $x \in Z^{(1)}$. This contradiction shows that the recurrent points form an invariant subset of $Z^{(2)}$, and since they are dense, $Z^{(2)}$ is invariant.

Let $J_{1}, J_{2}$ be ideals in $\mathbf{Z}^{+} \times_{\phi} C_{0}(X)$ associated with the constant sequences $\left(Z^{(1)}\right),\left(Z^{(2)}\right)$, respectively. Now $J_{1} J_{2} \subset J_{1} \cap J_{2} \sim\left(Z^{(1)} \cup Z^{(2)}\right)=(Z)$, and since $J$ is prime either $J_{1} \subset J$ or $J_{2} \subset J$. That is, either $Z \subset Z^{(1)}$ or $Z \subset Z^{(2)}$. But $Z^{(2)}=\left(Z \backslash Z^{(1)}\right)^{-} \subset Z \backslash\left(\operatorname{int} Z^{(1)}\right)$, so $Z^{(2)}$ is properly contained in $Z$ as $\operatorname{int} Z^{(1)} \neq$ $\varnothing$. Thus it must be that $Z^{(1)}=Z$, and by Proposition II. $8(Z, \varphi)$ is topologically transitive, and any proper closed invariant subset must be nowhere dense.

III.8. PROPOSITION. Let $(X, \phi)$ be a dynamical system such that $\phi$ is free. Let $Z \subset X$ be closed, invariant and transitive (so $Z=0^{+} \overline{\left(x_{0}\right)}$, some $x_{0} \in X$ ). then the ideal $\mathrm{J} \sim(Z)$ is prime in $\mathrm{Z}^{+} \times_{\phi} C_{0}(X)$.

PROOF. Let $J_{1}, J_{2}$ be closed, two sided ideals in $\mathbf{Z}^{+} \times_{\phi} C_{0}(X)$ such that $J_{1} J_{2} \subset J$. By Theorem III.5 there are sequences $\left(Y_{n}^{(1)}\right)_{n=0}^{\infty} \sim J_{1}$ and $\left(Y_{n}^{(2)}\right)_{n=0}^{\infty} \sim J_{2}$. Fix $n_{1}, n_{2} \in \mathbf{Z}^{+}$and let $f, g \in C_{0}(X)$ be such that $\{x: f(x)=0\}=Y_{n_{1}}^{(1)},\{x: g(x)=$ $0\}=Y_{n_{2}}^{(2)}$. Then $U^{n_{1}} f \in J_{1}, U^{n_{2}} g \in J_{2}$, and so for any $k \in \mathbf{Z}^{+}, U^{n_{1}} f U^{k} U^{n_{2}} g=$ $U^{n_{1}+n_{2}+k} f \circ \phi^{k+n_{2}} g \in J$. This means $f \circ \phi^{k+n_{2}} g$ vanishes on $Z$. Suppose there is a transitive point $x_{1}$ (i.e., $O+\overline{\left(x_{1}\right)}=Z$ ) such that $g\left(x_{1}\right) \neq 0$. Then $f \circ \phi^{k+n_{2}}\left(x_{1}\right)=0$, so $\phi^{k+n_{2}}\left(x_{1}\right) \in Y_{n_{1}}^{(1)}$ for all $k \geq 0$. Since $x_{1}$ is recurrent, $\mathcal{O}^{+} \overline{\left(x_{1}\right)}=\mathcal{O}^{+}\left(\phi^{n_{2}} \overline{\left(x_{1}\right)}\right)=$ $Z$, and so $Z \subset Y_{n_{1}}^{(1)}$. On the other hand, if $g(x)=0$ for every transitive point $x \in Z$, since by Proposition II. 8 the transitive points are dense, it follows $g$ vanishes on $Z$, and so $Z \subset Y_{n_{2}}^{(2)}$. Thus, for every $n$, either $Z \subset Y_{n}^{(1)}$ or $Z \subset Y_{n}^{(2)}$, and hence, either $Z \subset Y_{n}^{(1)}$ for infinitely many $n$ or else $Z \subset Y_{n}^{(2)}$ for finitely many $n$. Say $Z \subset Y_{n}^{(1)}$ for infinitely many $n$. As $Y_{n+1}^{(1)} \subset Y_{n+1}^{(1)} \cup \phi\left(Y_{n+1}^{(1)}\right) \subset Y_{n}^{(1)}$, it follows $Z \subset Y_{n}^{(1)}$ for all $n$, and hence $J_{1} \subset J$. In the other case we conclude $J_{2} \subset J$. Thus $J$ is prime.

III.9. Let $(X, \phi)$ be a dynamical system, and set $\mathfrak{A}=\mathbf{Z}^{+} \times_{\phi} C_{0}(X)$. A closed, two sided ideal $J \subset \mathfrak{A}$ is called right primitive if $\mathfrak{A} / J$ is a (right) primitive algebra; in other words, $\mathfrak{A} / \mathcal{J}$ can be faithfully represented as a strictly irreducible (or strictly transitive) right $\mathcal{M}$-module, for some vector space $\mathcal{M}$. $\mathcal{M}$ is isomorphic to $\mathfrak{A} / R$ for some maximal right ideal $R \subset \mathfrak{A}$, and $\mathfrak{A} / R$ is a Banach space under a suitable norm, and so $\mathfrak{A} / \mathcal{J}$ is represented as an algebra of continuous operators on a Banach space (acting on the right) (see $[12,2.4 .7]$ ).

III.10. The proof of the next proposition is deferred until V.5.

PROPOSITION. Let $(X, \phi)$ be a dynamical system, and $Z \subset X$ a compact minimal invariant subset. If $J \subset \mathbf{Z}^{+} \times_{\phi} C_{0}(X)$ is the closed two sided ideal associated with the constant sequence $(Z)$, then $J$ is right primitive.

III.11. Let $(X, \phi)$ be a dynamical system with $\phi$ freely acting. We summarize the results from this section regarding the maximal, primitive and prime ideals of $\mathbf{Z}^{+} \times_{\phi} C_{0}(X)$.

THEOREM. (i) There is a one-to-one correspondence between the maximal two sided ideals $J$ of $\mathbf{Z}^{+} \times_{\phi} C_{0}(X)$ and the points of $X$ as follows: $J$ corresponds to $x \in X$ iff $J \sim\left(Y_{n}\right)_{n=0}^{\infty}$, with $Y_{0}=\{x\}, Y_{n}=\varnothing, n \geq 1$. 
(ii) There is a one-to-one mapping from the compact minimal invariant subsets $Z \subset X$ into the (right) primitive ideals $J$ of $\mathbf{Z}^{+} \times_{\phi} C_{0}(X)$ as follows: $Z \mapsto J$ if

$$
J=\left\{F \in \mathbf{Z}^{+} \times_{\phi} C_{0}(X): P_{n}(F) \mid Z=0, n=0,1,2, \ldots\right\} .
$$

(iii) There is a one-to-one correspondence between prime ideals $J \subset \mathbf{Z}^{+} \times_{\phi} C_{0}(X)$ which are not maximal, and closed, invariant transitive sets $Z \subset X$ as follows: $J \sim(Z)$ iff

$$
J=\left\{F \in \mathbf{Z}^{+} \times_{\phi} C_{0}(X): P_{n}(F) \mid Z=0, n=0,1, \ldots\right\} .
$$

III.12. We conclude this section with some remarks concerning the hull-kernel topology on the various spaces of ideals. Effros and Hahn [5] showed that if $\phi$ is a freely acting homeomorphism of a locally compact space $X$, there is a one-to-one correspondence between the primitive ideals of the $C^{*}$-crossed product $\mathbf{Z} \times{ }_{\phi} C_{0}(X)$ and the set of orbit closures in $X$. In their case, the orbit was two sided: $O(x)=$ $\left\{\phi^{n}(x): n \in \mathbf{Z}\right\}$. Define a map $p$ from $X$ to the collection of closed orbits by $p(x)=O \overline{(x)}$. Then $p(X)$, provided with the quotient topology, is homeomorphic with $\operatorname{Prim}\left(\mathbf{Z} \times{ }_{\phi} C_{0}(X)\right)$.

The results for $\mathbf{Z}^{+} \times_{\phi} C_{0}(X)$ are similar, through somewhat more complicated to state. Let $\phi$ be freely acting. The correspondence described in III.11(i) is a homeomorphism of $X$ with the space of maximal, two sided ideals (i.e., the strong structure space) of $\mathrm{Z}^{+} \times_{\phi} C_{0}(X)$. This was proved in [11, IV.8] and also follows easily from the results of this section. Next consider the hull-kernel topology on the collection of prime ideals which are not maximal. Let $S=\left\{J_{i}\right\}, J_{i} \sim\left(Z^{(i)}\right)$, be a collection of prime ideals which are not maximal. A nonmaximal prime ideal $\mathrm{J}$, $J \sim(Z)$, belongs to the closure of $S$ iff $J \supset \bigcap J_{i}$, iff $Z \subset\left(\bigcup Z^{(i)}\right)^{-}$.

\section{The conjugate space.}

IV.1. Let $(X, \phi)$ be a dynamical system and let $\xi, \eta \in l_{1}^{+}$; the map $F \in \mathbf{Z}^{+} \times_{\phi}$ $C_{0}(X) \rightarrow \Lambda_{x}(F)=\left(\Pi_{x}(F) \xi, \eta\right)$ defines a continuous linear functional on $\mathbf{Z}^{+} \times_{\phi}$ $C_{0}(X)$.

LEMMA. Let $J \subset \mathbf{Z}^{+} \times_{\phi} C_{0}(X)$ be a closed two sided ideal, $J \sim(Z)$, where $Z \subset X$ is closed, invariant and $\phi^{-1}(Z)=Z$. Then $\Lambda_{x} \in J^{\perp}$ iff $x \in Z$.

Proof. Suppose $x \in Z$ and $F=\sum_{n=0}^{\infty} U^{n} f_{n} \in J$. Then $f_{n} \mid Z=0$ for all $n$, so $\Pi_{x}(F)=0$. Thus $\Lambda_{x} \in J^{\perp}$.

Conversely, let $\Lambda_{x} \in J^{\perp}$; if $\Lambda_{x} \neq 0$, then $\Lambda_{x}\left(U^{n} g\right) \neq 0$ for some $n \in \mathbf{Z}^{+}$and $g \in C_{0}(X)$. If $\xi=\left(\xi_{n}\right)_{n=0}^{\infty}, \eta=\left(\eta_{n}\right)_{n=0}^{\infty}$, then $\Lambda_{x}\left(U^{n} g\right)=\sum_{i=0}^{\infty} g \circ \phi^{i}(x) \xi_{i} \bar{\eta}_{n+i}$. Adjusting $g$ by a scalar, we may assume $\Lambda_{x}\left(U^{n} g\right)=1$. Choose $N$ large enough so that both

$$
\left|\sum_{i=0}^{N} g \circ \phi^{i}(x) \xi_{i} \bar{\eta}_{n+i}\right|>\frac{2}{3} \text { and } \sum_{i=N+1}^{\infty}\left|\xi_{i} \bar{\eta}_{n+i}\right|<\frac{1}{3\|g\|}
$$

Now if $x \notin Z, \phi^{i}(x) \notin Z$ for all $i=0,1, \ldots$, and so there exists $f \in C_{0}(X)$ such that $f \mid Z=0, f\left(\phi^{i}(x)\right)=g\left(\phi^{i}(x)\right), i=0,1, \ldots, N$, and $\|f\| \leq\|g\|$. On the one 
hand, since $\Lambda_{x} \in J^{\perp}$ and $U^{n} f \in J, \Lambda_{x}\left(U^{n} f\right)=0$. However,

$$
\begin{aligned}
\left|\Lambda_{x}\left(U^{n} f\right)\right| & =\left|\sum_{i=0}^{\infty} f \circ \phi^{i}(x) \xi_{i} \bar{\eta}_{n+i}\right| \\
& \geq\left|\sum_{i=0}^{N} f \circ \phi^{i}(x) \xi_{i} \bar{\eta}_{n+i}\right|-\left|\sum_{i=N+1}^{\infty} f \circ \phi^{i}(x) \xi_{i} \bar{\eta}_{n+i}\right| \\
& \geq\left|\sum_{i=0}^{N} g \circ \phi^{i}(X) \xi_{i} \bar{\eta}_{n+i}\right|-\|f\| \sum_{i=N+1}^{\infty}\left|\xi_{i} \eta_{n+i}\right| \\
& >\frac{2}{3}-\|g\| \frac{1}{3\|g\|}=\frac{1}{3} .
\end{aligned}
$$

This contradiction shows that $x \in Z$, as asserted.

Let $F \in K\left(\mathbf{Z}^{+}, C_{0}(X)\right), F=\sum_{k=0}^{N} U^{k} f_{k}$. The element $F^{\prime}=\sum_{k=0}^{N} U^{k} f_{k}^{\prime}$, where $f_{k}^{\prime}=f_{k} \mid Z$, belongs to $K\left(\mathbf{Z}^{+}, C_{0}(Z)\right.$ ), whose norm (in $\mathbf{Z}^{+} \times_{\phi} C_{0}(Z)$ ) is given by $\sup _{x \in Z}\left\|\Pi_{x}\left(F^{\prime}\right)\right\|$, which is no greater than $\sup _{x \in X}\left\|\Pi_{x}(F)\right\|=\|F\|$. This mapping extends to a map $\Pi: \mathbf{Z}^{+} \times_{\phi} C_{0}(X) \rightarrow \mathbf{Z}^{+} \times_{\phi} C_{0}(Z)$, and $\|\Pi(F)\| \leq\|F\|$, $F \in \mathbf{Z}^{+} \times_{\phi} C_{0}(X)$. As $J=\operatorname{ker} \Pi, \Pi\left(\mathbf{Z}^{+} \times_{\phi} C_{0}(X)\right)$ and $\mathbf{Z}^{+} \times_{\phi} C_{0}(X) / J$ are algebraically isomorphic. If $Z$ is just invariant $(\phi(Z) \subset Z$ or even $\phi(Z)=Z)$, it is an open question whether $\Pi$ is surjective.

COROLlARY. Under the hypotheses of the Lemma, the map $\Pi$ is surjective, and $\mathbf{Z}^{+} \times_{\phi} C_{0}(X) / J$ and $\mathbf{Z}^{+} \times_{\phi} C_{0}(Z)$ are isomorphic as Banach algebras.

PROOF. First we claim $J^{\perp}$ is the closed linear span of $\left\{\Lambda_{x}=\Lambda_{x}(\xi, \eta): x \in\right.$ $\left.Z, \xi, \eta \in l_{2}^{+}\right\}$. Let $K$ be this closed subspace, and let $\Lambda_{0} \in J^{\perp}$ and suppose $\Lambda_{0} \notin K$. By the Hahn-Banach Theorem, there is an $F \in \mathbf{Z}^{+} \times_{\phi} C_{0}(X)$ such that $\operatorname{Re}\left(\Lambda_{0}(F)\right)>0, \operatorname{Re}(\Lambda(F)) \leq 0$ for all $\Lambda \in K$. But as $K$ is a subspace, it must be that $\Lambda(F)=0$ for all $\Lambda \in K$. So $\Pi_{x}(F)=0, x \in Z$, and hence, by the Lemma, $F \in J$. But then $\Lambda_{0}(F)=0$.

The quotient norm is given by

$$
\|F+J\|=\inf _{G \in J}\|F+G\|=\sup _{\substack{\Lambda \in J^{\perp} \\\|\Lambda\| \leq 1}}|\Lambda(F)| .
$$

By the claim, the linear functionals $\Lambda_{x}(x \in Z)$ are total in $J^{\perp}$, so that

$$
\|F+J\|=\sup _{\left\|\Lambda_{x}\right\| \leq 1, x \in Z}\left|\Lambda_{x}(F)\right| .
$$

But the right side is precisely the norm of $\Pi(F)$ in $\mathbf{Z}^{+} \times_{\phi} C_{0}(Z)$. Thus $\Pi$ maps onto $\mathbf{Z}^{+} \times_{\phi} C_{0}(Z)$, and $\mathbf{Z}^{+} \times_{\phi} C_{0}(X) / J$ and $\mathbf{Z}^{+} \times_{\phi} C_{0}(Z)$ are isomorphic as Banach algebras.

IV.2. Let $(X, \phi)$ be a dynamical system, and set $\mathfrak{A}=\mathbf{Z}^{+} \times_{\phi} C_{0}(X)$. Let $\mathfrak{A}^{*}$ denote the conjugate space of $\mathfrak{A}$ (the continuous linear functionals on $\mathfrak{A}$ ).

A. Lemma. Let $F \in \mathfrak{A}, F=\sum_{n=0}^{\infty} U^{n} f_{n}$. Then $\lim _{n \rightarrow \infty}\left\|f_{n}\right\|=0$.

Proof. Since $K\left(\mathbf{Z}^{+}, C_{0}(X)\right)$ is dense in $\mathfrak{A}$, given $F \in \mathfrak{A}$ and $\varepsilon>0$ there exists $G \in K\left(\mathbf{Z}^{+}, C_{0}(X)\right)$ such that $\|F-G\|<\varepsilon$. By $[\mathbf{1 1}$, II.7] the norm of an element 
of $\mathfrak{A}$ is not less than the norms of any of its Fourier coefficients. If $G=\sum_{j=0}^{N} U^{i} g_{i}$, then for $n>N$ the $n$th Fourier coefficient of $F-G$ is $f_{n}$, and $\left\|f_{n}\right\| \leq\|F-G\|<\varepsilon$.

By the Lemma, $\mathfrak{A}$ is a subspace of the Banach space $c_{0}\left(C_{0}(X)\right)$ of sequences $\left(f_{n}\right)_{n=0}^{\infty}$ of functions in $C_{0}(X)$, with $\lim \left\|f_{n}\right\|=0$. Thus,

$$
l_{1}\left(C_{0}(X)\right) \subset \mathfrak{A} \subset c_{0}\left(C_{0}(X)\right) .
$$

So, $l_{\infty}(\mathcal{M}(X)) \supset \mathfrak{A}^{*} \supset l_{1}(\mathcal{M}(X))$, where $\mathcal{M}(X)$ is the space of complex regular Borel measures on $X$ with finite total variation. In other words, to every $\Gamma \in \mathfrak{A}^{*}$ there corresponds a unique sequence $\left(\mu_{n}\right)_{n=0}^{\infty}, \mu_{n} \in \mathcal{M}(X)$, such that for every $F=\sum_{n=0}^{N} U_{n} f_{n} \in K\left(\mathbf{Z}^{+}, C_{0}(X)\right)$

$$
\langle\Gamma, F\rangle=\sum_{n=0}^{N} \int f_{n} d \mu_{n} .
$$

Also, $\|\Gamma\| \geq \sup _{n}\left\|\mu_{n}\right\|$. It will be convenient to write $\Gamma=\left(\mu_{n}\right)_{n=0}^{\infty}$. (Note: from now on we will use the bracket $\langle\cdot, \cdot\rangle$ notation for linear functionals in place of the notation of (IV.1).)

B. REMARKS. (i) If $F \in \mathfrak{A}$ with Fourier series $F=\sum_{n=0}^{\infty} U^{n} f_{n}$, and if $\Gamma_{x} \in \mathfrak{A}^{*}$ is identified with the sequence $\left(\xi_{n} \delta_{x}\right)_{n=0}^{\infty}$ where $\delta_{x}$ is the point mass at $X$, the series $\sum_{n=0}^{\infty} \xi_{n} f_{n}(x)$ may not necessarily converge, just as the partial sum of the Fourier series $\sum_{n=0}^{\infty} U^{n} f_{n}$ need not converge to $F$. (However, both are Cesàro summable.)

(ii) As an example, let $\left(\xi_{n}\right)_{n=0}^{\infty} \in l_{2}^{+}$. Then $\Gamma_{x}=\left(\xi_{n} \delta_{x}\right)_{n=0}^{\infty} \in \mathfrak{A}^{*}$, for $\left\langle\Gamma_{x}, F\right\rangle=$ $\left(\Pi_{x}(F) \eta, \xi\right)$, where $\eta=(1,0,0, \ldots) \in l_{2}^{+}$, and $\xi=\left(\bar{\xi}_{n}\right)_{n=0}^{\infty}$.

\section{Irreducible Banach modules.}

V.1. Let $(X, \phi)$ be a dynamical system, and $R \subset \mathfrak{A}=\mathbf{Z}^{+} \times_{\phi} C_{0}(X)$ a closed right ideal. $R$ is thus a closed linear subspace of $\mathfrak{A}$ which is a right $C_{0}(X)$-module and invariant under right multiplication by $U$; conversely, any closed linear subspace $R \subset \mathfrak{A}$ with these properties is a closed right ideal.

If $\mathcal{R}$ is a subset of $\mathfrak{A}$, denote by $\mathcal{R}^{\perp}=\left\{\Gamma \in \mathfrak{A}^{*}:\langle\Gamma, F\rangle=0, F \in R\right\}$. If $R$ is a right ideal of $\mathfrak{A}$, then $R^{\perp}$ is a (left) $C_{0}(X)$-module under $\langle f \Gamma, F\rangle=\langle\Gamma, F f\rangle$ $(F \in \mathfrak{A})$. Furthermore, $R^{\perp}$ is invariant under the operator $T$ defined to be the adjoint of right multiplication by $U:\langle T \Gamma, F\rangle=\langle\Gamma, F U\rangle$.

Conversely, if $S \subset \mathfrak{A}^{*}$ is a closed linear subspace which is (i) a left $C_{0}(X)$ module, and (ii) invariant under $T$, then $S_{\perp}=\{F \in \mathfrak{A}:\langle\Gamma, F\rangle=0, \Gamma \in S\}$ is a closed right ideal of $\mathfrak{A}$.

As observed in [11, IV.3], the endomorphism $\alpha$ of $C_{0}(X), \alpha(f)=f \circ \phi$, admits an extension to an endomorphism of the Banach algebra $\mathfrak{A}$; this extension will also be denoted by $\alpha$. For $F=\sum_{n=0}^{N} U^{n} f_{n} \in K\left(\mathbf{Z}^{+}, C_{0}(X)\right), \alpha(F)=\sum_{n=0}^{N} U^{n} f_{n} \circ \phi$. One computes that for $F \in K\left(\mathbf{Z}^{+}, C_{0}(X)\right), \alpha(U F)=U(\alpha(F))=F U$, and so these equalities hold for $F \in \mathfrak{A}$ as well. Hence if $S$ denotes the adjoint of left multiplication by $U$, and $+\alpha$ the adjoint of $\alpha$,

$$
T={ }^{+} \alpha S=S^{+} \alpha .
$$

If $R \subset \mathfrak{A}$ is a right ideal, $\mathcal{R}^{\perp}$ will not, in general, be invariant under either ${ }^{+} \alpha$ or $S$.

V.2. Let $\lambda$ be a complex number, $0 \leq|\lambda|<1$. Since $\left(\lambda^{n}\right)_{n=0}^{\infty} \in l_{2}^{+}$, it follows from Remark (ii) of IV.2 that for each $x \in X$ the linear functional corresponding to 
the sequence $\left(\lambda^{n} \delta_{x}\right)_{n=0}^{\infty}$ is bounded, and hence there is a unique positive constant $c(\lambda, x)$ such that $c(\lambda, x)\left(\left(\lambda^{n} \delta_{x}\right)_{n=0}^{\infty}\right)$ has norm one. Now as ${ }^{+} \alpha$ has norm one,

$$
{ }^{+} \alpha\left(c(\lambda, x)\left(\lambda^{n} \delta_{x}\right)_{n=0}^{\infty}\right)=c(\lambda, x)\left(\lambda^{n} \delta_{\phi(x)}\right)_{n=0}^{\infty}
$$

has norm at most one. The same can be said about $c(\lambda, x)\left(\lambda^{n} \delta_{\phi^{N}(x)}\right)_{n=0}^{\infty}$ for any $N$, and hence $\left\|c(\lambda, x)\left(\lambda^{n} \delta_{y}\right)_{n=0}^{\infty}\right\| \leq 1$ for any $y \in \mathcal{O}^{+} \overline{(x)}$. Thus $c(\lambda, y) \geq c(\lambda, x)$. Suppose now that $(X, \phi)$ is a compact, minimal dynamical system; then $x \in \mathcal{O}^{+} \overline{(y)}$, so $c(\lambda, x) \geq c(\lambda, y)$. It follows that $c(\lambda, x)=c_{\lambda}$ depends only on $\lambda$. Let $\Gamma_{x}^{(\lambda)}=$ $c_{\lambda}\left(\lambda^{n} \delta_{x}\right)_{n=0}^{\infty}$.

If $S$ is the operator defined in V.1, $\Gamma \in \mathfrak{A}^{*}, \Gamma=\left(\mu_{n}\right)_{n=0}^{\infty}$, then $S \Gamma=\left(\nu_{n}\right)_{n=0}^{\infty}$, where $\nu_{n}=\mu_{n+1}, n \geq 0$. In particular, $S \Gamma_{x}^{(\lambda)}=\lambda \Gamma_{x}^{(\lambda)}, x \in X$. Let $S$ be the closed linear subspace of $\mathfrak{A}^{*}$ generated by the linear functionals $\left\{\Gamma_{x}^{(\lambda)}: x \in X\right\}$. As every complex measure in $\mathcal{M}(X)$ is a weak-* limit of linear combinations of point masses, $S=\left\{\Gamma \in \mathfrak{A}^{*}:\langle\Gamma, F\rangle=\int_{X}\left\langle\Gamma_{x}^{(\lambda)}, F\right\rangle d \mu(x)\right.$, for some $\left.\mu \in \mathcal{M}(X)\right\}$. Because the set $\left\{\Gamma_{x}^{(\lambda)}: x \in X\right\}$ is invariant under ${ }^{+} \alpha$ and $S$, it follows that $S$ is invariant under ${ }^{+}{ }_{\alpha}$ and $S$, hence invariant under $T$.

LEMMA. Let $R^{(\lambda)}=S_{\perp}=\left\{F \in \mathfrak{A}:\left\langle\Gamma_{x}^{(\lambda)}, F\right\rangle=0\right.$ for all $\left.x \in X\right\}$. Then $R^{(\lambda)}$ is a closed, $\alpha$-invariant right ideal in $\mathfrak{A}$. Furthermore, the unit ball of $S$ is the closed, balanced convex hull of $\left\{\Gamma_{x}^{(\lambda)}: x \in X\right\}$.

ProOF. It remains only to be noted that $S$ is a (left) $C(X)$-module, which is clear.

COROLlaRY. For $G \in \mathfrak{A}$, the quotient norm in $\mathfrak{A} / \mathbb{R}^{(\lambda)}$,

$$
\begin{aligned}
\left\|G+R^{(\lambda)}\right\| & =\inf \left\{\|G+H\|: H \in R^{(\lambda)}\right\}=\sup \{|\langle\Gamma, G\rangle|: \Gamma \in S\} \\
& =\sup \left\{\left|\left\langle\Gamma_{x}^{(\lambda)}, G\right\rangle\right|: x \in X\right\} .
\end{aligned}
$$

V.3. If $R$ is a closed right ideal of a Banach algebra $\mathfrak{A}$, then $\mathfrak{A} / \mathbb{R}$ is a right $\mathfrak{A}$-module under right multiplication, and by a standard calculation (e.g. $[\mathbf{1 2}, \mathrm{p}$. 50]),

$$
\|G F+R\| \leq\|G+R\|\|F\|
$$

Let $\mathfrak{A}, R^{(\lambda)}$ be as in V.2 and define a map $\Phi_{\lambda}: \mathfrak{A} / R^{(\lambda)} \rightarrow C(X)$ by the formula

$$
\Phi_{\lambda}\left(F+R^{(\lambda)}\right)(x)=\left\langle\Gamma_{x}^{(\lambda)}, F\right\rangle, \quad F \in \mathfrak{A} .
$$

The map is well defined, and by Corollary V.2 it is an isometry. As $C(X) \subset \mathfrak{A}$ and $\Phi_{\lambda}\left(f+R^{(\lambda)}\right)=c_{\lambda} f, \Phi_{\lambda}$ is onto. Also, observe

$$
\begin{aligned}
\Phi_{\lambda}\left(F f+R^{(\lambda)}\right)(x) & =\left\langle\Gamma_{x}^{(\lambda)}, F f\right\rangle=\left\langle\Gamma_{x}^{(\lambda)}, F\right\rangle f(x) \\
& =\Phi_{\lambda}\left(F+R^{(\lambda)}\right)(x) f(x) ; \\
\Phi_{\lambda}\left(F U+R^{(\lambda)}\right)(x) & =\left\langle T \Gamma_{x}^{(\lambda)}, F\right\rangle=\left\langle S^{+} \alpha\left(\Gamma_{x}^{(\lambda)}\right), F\right\rangle=\left\langle S \Gamma_{\phi(x)}^{(\lambda)}, F\right\rangle \\
& =\left\langle\lambda \Gamma_{\phi(x)}^{(\lambda)}, F\right\rangle=\lambda \Phi_{\lambda}\left(F+R^{(\lambda)}\right) \circ \phi(x) .
\end{aligned}
$$

Thus, $\Phi$ implements an $\mathfrak{A}$-module isomorphism of $\mathfrak{A} / \mathbb{R}^{(\lambda)}$ and $C(X)$; under this isomorphism, an element $F=\sum_{n=0}^{N} U^{n} f_{n}$ acts on $C(X)$ on the right by

$$
[g] F=\left[\sum_{n=0}^{N} \lambda^{n} g \circ \phi^{n} f_{n}\right], \quad g \in C(X) .
$$


Since $\|[g] F\| \leq\|g\|\|F\|$, we obtain, taking $g \equiv 1$,

$$
\left\|\sum_{n=0}^{N} \lambda^{n} f_{n}\right\| \leq\|F\| .
$$

COROLLARY. $c_{\lambda}=1$; in other words, the linear functional $\Gamma_{x}^{(\lambda)}=\left(\lambda^{n} \delta_{x}\right)_{n=0}^{\infty}$ has norm one, $0 \leq|\lambda|<1$.

PROOF. Let $F=\sum_{n=0}^{N} U^{n} f_{n} ;\left|\left\langle\left(\lambda^{n} \delta_{x}\right)_{n=0}^{\infty}, F\right\rangle\right|=\left|\sum_{n=0}^{N} \lambda^{n} f_{n}(x)\right| \leq\|F\|$ by $(*)$, so $\left\|\left(\lambda^{n} \delta_{x}\right)_{n=0}^{\infty}\right\| \leq 1$. But $\left|\left\langle\left(\lambda^{n} \delta_{x}\right)_{n=0}^{\infty}, f\right\rangle\right|=|f(x)|=\|f\|$ if $f \in C_{0}(X)$ achieves its supremum at the point $x$. Thus $\left\|\left(\lambda^{n} \delta_{x}\right)_{n=0}^{\infty}\right\|=1$.

REMARK. The linear functionals $\Gamma_{x}^{(\lambda)}$ are defined for all $|\lambda|<1$. For $|\lambda|=1$, define $\Gamma_{x}^{(\lambda)}$ on $K\left(\mathbf{Z}^{+}, C_{0}(X)\right)$ by $\left\langle\Gamma_{x}^{(\lambda)}, F\right\rangle=\sum_{n=0}^{N} \lambda^{n} f_{n}(x), F=\sum_{n=0}^{N} U^{n} f_{n}$. Now $(*)$ implies, for every $0<r<1$,

$$
\left|\sum_{n=0}^{N} \lambda^{n} r^{n} f_{n}(x)\right| \leq\|F\| .
$$

Taking the limit as $r \uparrow 1$, we obtain $\left|\left\langle\Gamma_{x}^{(\lambda)}, F\right\rangle\right| \leq\|F\|$. As $K\left(\mathbf{Z}^{+}, C(X)\right) \subset \mathfrak{A}$ is dense, $\Gamma_{x}^{(\lambda)}$ has a unique extension to a (norm one) linear functional on $\mathfrak{A}$, again denoted $\Gamma_{x}^{(\lambda)}$. Of course this does not imply that for $F \in \mathfrak{A}$ arbitrary, with Fourier series $\sum_{n=0}^{\infty} U^{n} f_{n}$, that $\sum_{n=0}^{\infty} \lambda^{n} f_{n}(x)$ converges; but it is summable by a summability kernel.

V.4. Let $(X, \phi)$ be a compact minimal dynamical system, and $R^{(\lambda)}$ the closed, $\alpha$-invariant right ideal $R^{(\lambda)}=\left\{\Gamma_{x}^{(\lambda)}: x \in X\right\} \perp$.

THEOREM. Let $0<|\lambda| \leq 1$. The action of $\mathfrak{A}=\mathbf{Z}^{+} \times_{\phi} C(X)$ on $C(X)$, which is determined by $[g] f=[g f],[g] U=\lambda[g \circ \phi], g, f \in C(X)$, is strictly transitive. In fact, the action of the subalgebra $K\left(\mathbf{Z}^{+}, C(X)\right)$ is strictly transitive on $C(X)$.

Proof. In V.3 it was shown that $\mathfrak{A}$ acts on $C(X)$ as described above, and $\|[g] F\| \leq\|g\|\|F\|, g \in C(X), F \in \mathfrak{A}$. To show strict transitivity, let $0 \neq g$, $h \in C(X)$ be given. By the minimality of $(X, \phi)$ there exists $N \in \mathbf{Z}^{+}$such that $\bigcup_{j=0}^{N} \phi^{-j}(\mathcal{O})=X$, where $\mathcal{O}=\{x: g(x) \neq 0\}$ (Lemma II.9). Thus,

$$
|g|^{2}+|\lambda|^{2}|g \circ \phi|^{2}+\cdots+|\lambda|^{2 N}\left|g \circ \phi^{N}\right|^{2}
$$

is strictly positive on $X$. Note that

$$
[g] U^{k}\left(\bar{\lambda}^{k} g \circ \phi^{k}\right)=\left[|\lambda|^{2 k}\left|g \circ \phi^{k}\right|^{2}\right] .
$$

Let

$$
f=\left(\sum_{k=0}^{N}|\lambda|^{2 k}\left|g \circ \phi^{k}\right|^{2}\right)^{-1} .
$$

Then $f \in C(X)$, and $[h]=[g]\left(\sum_{k=0}^{N} U^{k} \bar{\lambda}^{k} g \circ \phi^{k}\right) f h$.

COROLLARY. The ideals $\mathcal{R}^{(\lambda)}(0<|\lambda| \leq 1)$ are maximal right ideals.

PROOF. Since $\mathfrak{A} / \mathcal{R}^{(\lambda)}$ and $C(X)$ with the action $[g] f=[g f],[g] U=\lambda[g \circ \phi]$ $(f, g \in C(X))$ are isomorphic as right $\mathfrak{A}$-modules, the maximality of $\mathcal{R}^{(\lambda)}$ follows from the Theorem and $[8,1.1 .3]$. 
It would be interesting to know if the ideals $R^{(\lambda)}(0<|\lambda| \leq 1)$ are the only maximal, $\alpha$-invariant right ideals in $\mathfrak{A}$. In conclusion we will show, under the additional assumption of unique ergodicity, that this is in fact the case.

V.5. The proof of Proposition III.10 can now be given. Let $(X, \phi)$ be a dynamical system and $Z \subset X$ be a compact minimal invariant subset, and let $J$ be the closed two sided ideal in $\mathbf{Z}^{+} \times_{\phi} C_{0}(X), J \sim(Z)$ (Notation III.6). Let $j: \mathbf{Z}^{+} \times_{\phi}$ $C_{0}(X) \rightarrow \mathbf{Z}^{+} \times_{\phi} C(Z)$ be the canonical map (see [11, II.12]), which is defined on the subalgebra $K\left(\mathbf{Z}^{+}, C_{0}(X)\right)$ by $j\left(\sum_{n=0}^{N} U^{n} f_{n}\right)=\sum_{n=0}^{N} U^{n} f_{n}^{\prime}$, where $f_{n}^{\prime}=$ $f_{n} \mid Z$. The image of $j$ contains $K\left(\mathbf{Z}^{+}, C(Z)\right)$, ker $j=J$, and $\|j(F)\| \leq\|F\|$ for $F \in \mathbf{Z}^{+} \times_{\phi} C_{0}(X)$. Since by Theorem V.4 there exist faithful right $\mathbf{Z}^{+} \times_{\phi} C(Z)$ modules such that the subalgebra $K\left(\mathbf{Z}^{+}, C(Z)\right)$ acts strictly transitively, it follows that $J$ is a (right) primitive ideal in $\mathbf{Z}^{+} \times_{\phi}(X)$.

V.6. Assume that the dynamical system $(X, \phi)$ is transitive and $X$ is compact. Let $\mathfrak{A}=\mathbf{Z}^{+} \times_{\phi} C(X)$ and $\mathfrak{A}_{0}$ be the subalgebra generated by $\{1, U\} . \mathfrak{A}_{0}$ is a commutative Banach algebra; it is the subalgebra of all $F \in \mathfrak{A}$ whose Fourier coefficients are all constant functions.

\section{PROPOSITION. $\mathfrak{A}_{0} \subset \mathfrak{A}$ is a maximal abelian subalgebra.}

ProOF. Let $F \in \mathfrak{A}$ be such that $F U=U F$. If $F=\sum_{n=0}^{\infty} U^{n} f_{n}$, then $f_{n} \circ \phi=f_{n}$. This means that $f_{n}$ is constant on each orbit $\mathcal{O}^{+}(x)$. Since $(X, \phi)$ is transitive, there exists $x_{0} \in X$ with $\mathcal{O}^{+}\left(\overline{x_{0}}\right)=X$. Thus $f_{n}$ is constant, $n=0,1, \ldots$, and $F \in \mathfrak{A}_{0}$.

V.7. The disk algebra is the commutative Banach algebra of continuous functions on the closed unit disk $\{z \in \mathbf{C}:|z| \leq 1\}$ which are holomorphic in the interior. For the next result, we need only assume $X$ is compact. As before, $\mathfrak{A}=\mathbf{Z}^{+} \times_{\phi} C(X)$ and $\mathfrak{A}_{0}$ is the closed subalgebra generated by $\{1, U\}$.

PROPOSITION. The mapping which takes an element $F \in \mathfrak{A}_{0}$ with Fourier series $\sum_{n=0}^{\infty} U^{n} c_{n}$ to the analytic function with power series $\sum_{n=0}^{\infty} c_{n} z^{n}$ is an isometric Banach algebra isomorphism of $\mathfrak{A}_{0}$ with the disk algebra.

PROOF. Since for any $F \in \mathfrak{A}_{0}$, the norms $\left\|\Pi_{x}(F)\right\|(x \in X)$ all coincide, $\mathfrak{A}_{0}$ is naturally isomorphic with $\mathbf{Z}^{+} \times_{\phi} C\left(X_{0}\right)$ where $X_{0}$ consists of a single point and $\phi_{0}$ is the identity map. Now $\mathbf{Z}^{+} \times_{\phi_{0}} C\left(X_{0}\right)$ is the disk algebra [11, Theorem III.2 (case $\left.k_{0}=1\right)$ ], and apply [11, Corollary II.10].

Note. It would not be hard to give a direct proof of the proposition.

V.8. Assume that $(X, \phi)$ is a compact minimal dynamical system which is uniquely ergodic. Let $\omega$ be the unique invariant probability measure on $X$. For $f \in$ $C(X)$, the averages $\left[f+f \circ \phi+\cdots+f \circ \phi^{n}\right] /(n+1)$ converge uniformly to a constant, which is $\int_{X} f d \omega\left[6\right.$, Theorem 2.3.5]. Define $Q_{n}: K\left(\mathbf{Z}^{+}, C(X)\right) \rightarrow$ $K\left(\mathbf{Z}^{+}, C(X)\right)$ by $Q_{n}(F)=\sum_{k=0}^{n} \alpha^{k}(F) /(n+1)$. Since $\alpha$ is a contraction, so is $Q_{n}$. If $F=\sum_{j=0}^{N} U^{j} f_{j}, Q_{n}(F)=\sum_{j=0}^{N} U^{j} Q_{n}\left(f_{j}\right)$, and since $\left\{Q_{n}\left(f_{j}\right)\right\}_{n=0}^{\infty}$ converges uniformly, $0 \leq j \leq N,\left\{Q_{n}(F)\right\}_{n=0}^{\infty}$ converges in the norm of $\mathfrak{A}=\mathbf{Z}^{+} \times_{\phi} C(X)$. Denote the limit of the sequence $\left\{Q_{n}(F)\right\}_{n=0}^{\infty}$ by $F^{\sharp}$. Now let $F \in \mathfrak{A}$ and $\varepsilon>0$ be given. If $G \in K\left(\mathbf{Z}^{+}, C(X)\right)$ is such that $\|F-G\|<\varepsilon$, and $M \in \mathbf{Z}^{+}$such that $n \geq M$ implies $\left\|Q_{n}(G)-G^{\sharp}\right\|<\varepsilon$, then

$$
\left\|Q_{n}(F)-G^{\sharp}\right\| \leq\left\|Q_{n}(F-G)\right\|+\left\|Q_{n}(G)-G^{\sharp}\right\|<2 \varepsilon,
$$


and so $\left\|Q_{n}(F)-Q_{m}(F)\right\|<4 \varepsilon, n, m \geq M$. As $\alpha\left(G^{\sharp}\right)=G^{\sharp}$, it follows

$$
\left\|\alpha^{k}\left(Q_{n}\right)(F)-Q_{n}(F)\right\|<4 \varepsilon, \quad k=0,1, \ldots
$$

Thus the sequence $\left\{Q_{n}(F)\right\}_{n=0}^{\infty}$ is Cauchy in $\mathfrak{A}$, and its limit is $\alpha$-invariant, and consequently belongs to the subalgebra $\mathfrak{A}_{0}$ generated by $\{1, U\}$. Denote the limit by $F^{\sharp}$.

PROPOSITION. The operator $\sharp: \mathfrak{A} \rightarrow \mathfrak{A}_{0}$ has the following properties:

(i) $\left\|F^{\sharp}\right\| \leq\|F\|$;

(ii) $\sharp$ is a linear projection;

(iii) $F^{\sharp}$ belongs to the closed convex hull of $\left\{\alpha^{n}(F)\right\}_{n=0}^{\infty}$;

(iv) If $F \in \mathfrak{A}$ and $G \in \mathfrak{A}_{0}$, then $(F G)^{\sharp}=F^{\sharp} G$, and $(G F)^{\sharp}=G F^{\sharp}$.

PROOF. (i) and (iii) have already been verified, while (ii) and (iv) are straightforward.

V.9. Let $(X, \phi)$ be as in V.6. If $R \subset \mathfrak{A}$ is an $\alpha$-invariant right ideal, set $R^{\sharp}=$ $\left\{F^{\sharp}: F \in R\right\}$. If $I \subset \mathfrak{A}_{0}$ is an ideal, set $\tilde{I}=\left\{F \in \mathfrak{A}:\left(\alpha^{n}(F) G\right)^{\sharp} \in I\right.$, for all $G \in \mathfrak{A}$, $n=0,1, \ldots\}$.

A. Proposition. (i) If $R$ is an $\alpha$-invariant closed right ideal in $\mathfrak{A}, R^{\sharp}$ is a closed ideal in $\mathfrak{A}_{0}$, and $R^{\sharp}=R \cap \mathfrak{A}_{0}$.

(ii) If $I$ is a closed ideal in $\mathfrak{A}_{0}, \tilde{I}$ is an $\alpha$-invariant right ideal in $\mathfrak{A}$, and $\tilde{I}$ is the unique maximal element of the set $\left\{J: J\right.$ an $\alpha$-invariant right ideal of $\left.\mathfrak{A}, J^{\sharp} \subset I\right\}$.

Proof. (i) By Proposition V.6(iv), $R^{\sharp}$ is an ideal in $\mathfrak{A}_{0}$. If $F \in R, F^{\sharp}$ is in the closed convex hull of $\left\{\alpha^{n}(F)\right\}_{n=0}^{\infty} \subset R$, so $F^{\sharp} \in R \cap \mathfrak{A}_{0}$. Thus $R^{\sharp} \subset R \cap \mathfrak{A}_{0}$. Since $\sharp$ is a projection, $R \cap \mathfrak{A}_{0}=\left(R \cap \mathfrak{A}_{0}\right)^{\sharp} \subset R^{\sharp}$.

(ii) By its definition, $\tilde{I}$ is an $\alpha$-invariant right ideal. Let $R$ be any $\alpha$-invariant right ideal such that $R^{\sharp} \subset J$. If $F \in R, \alpha^{n}(F) G \in R$ for all $n \geq 0, G \in \mathfrak{A}$. So $\left(\alpha^{n}(F) G\right)^{\sharp} \in R^{\sharp} \subset I$. It follows that $R \subset \tilde{I}$.

B. Proposition. If $\mathcal{M}_{0} \subset \mathfrak{A}_{0}$ is a maximal ideal, then $\tilde{\mathcal{M}}_{0} \subset \mathfrak{A}$ is a maximal $\alpha$-invariant right ideal.

Proof. Observe that $\left(\tilde{\mathcal{M}}_{0}\right)^{\sharp}=\mathcal{M}_{0}$, and hence $\tilde{\mathcal{M}}_{0}$ is proper. Let $R \subset \tilde{\mathcal{M}}_{0}$ be an $\alpha$-invariant right ideal. Now $R^{\sharp}$ is proper, for if $R^{\sharp}=\mathfrak{A}_{0}$, then $R \cap \mathfrak{A}_{0}=\mathfrak{A}_{0}$, so $1 \in R$, a contradiction. By the maximality of $\mathcal{M}_{0}, R^{\sharp}=\left(\tilde{\mathcal{M}}_{0}\right)^{\sharp}=\mathcal{M}_{0}$. By Proposition $\mathrm{A}$ (ii), $\mathcal{M}_{0}=R$, and hence $\mathcal{M}_{0}$ is a maximal $\alpha$-invariant right ideal.

C. COROllaRY. If $R \subset \mathfrak{A}$ is any $\alpha$-invariant right ideal, $R \subset\left(R^{\sharp}\right)^{\sim}$. Hence if $R$ is a maximal $\alpha$-invariant right ideal, $R=\left(R^{\sharp}\right)^{\sim}$.

D. COROLlaRY. If $R \subset \mathfrak{A}$ is a maximal $\alpha$-invariant right ideal, then $R^{\sharp} \subset \mathfrak{A}_{0}$ is a maximal ideal.

PRoOF. Let $\mathcal{M}_{0} \supset R^{\sharp}$ be a maximal ideal in $\mathfrak{A}_{0}$. By Proposition $B, \tilde{\mathcal{M}}_{0}$ is a maximal $\alpha$-invariant right ideal, and since $\tilde{\mathcal{M}}_{0} \supset\left(R^{\sharp}\right)^{\sim}=R$ (Corollary C), it follows that $\tilde{M}_{0}=R$. Therefore $\mathcal{M}_{0}=\left(\tilde{\mathcal{M}}_{0}\right)^{\sharp}=R^{\sharp}$ is maximal.

E. SUMMARY. There is a one-to-one correspondence between the set $R_{\alpha}$ of maximal $\alpha$-invariant right ideals of $\mathfrak{A}$, and the set $\underline{M}$ of maximal ideals of $\mathfrak{A}_{0}$, given by

$$
R \in \underline{R_{\alpha}} \mapsto R^{\sharp} \in \underline{M} \text { and } \mathcal{M} \in \underline{M} \mapsto \tilde{\mathcal{M}} \in \underline{R_{\alpha}} .
$$

V.10. Assume $(X, \phi)$ is compact, minimal, free and uniquely ergodic. 
LEMMA. Each $\gamma \in \mathfrak{A}_{0}^{*}$ extends to a unique $+\alpha$-invariant element $\Gamma$ of $\mathfrak{A}^{*}$.

Proof. Let $\Gamma, \Gamma^{\prime} \in \mathfrak{A}^{*}$ be ${ }^{+} \alpha$-invariant, and suppose $\langle\Gamma, G\rangle=\left\langle\Gamma^{\prime}, G\right\rangle$ for all $G \in$ $\mathfrak{A}_{0}$. If $F \in \mathfrak{A},\langle\Gamma, F\rangle=\left\langle\Gamma, Q_{n}(F)\right\rangle$ by invariance, hence $\langle\Gamma, F\rangle=\left\langle\Gamma, F^{\sharp}\right\rangle$ (cf. V.6). Since the same holds for $\Gamma^{\prime}$, we have $\langle\Gamma, F\rangle=\left\langle\Gamma, F^{\sharp}\right\rangle=\left\langle\Gamma^{\prime}, F^{\sharp}\right\rangle=\left\langle\Gamma, F^{\sharp}\right\rangle=\left\langle\Gamma^{\prime}, F\right\rangle$. Thus, the restriction map from the subspace of ${ }^{+} \alpha$-invariant elements of $\mathfrak{A}^{*}$ to $\mathfrak{A}_{0}^{*}$ is injective. To see that it is surjective, let $\gamma \in \mathfrak{A}_{0}^{*}$; by the Hahn-Banach Theorem there is a $\Gamma_{1} \in \mathfrak{A}^{*}$ such that $\Gamma_{1} \mid \mathfrak{A}_{0}=\gamma$. Define $\Gamma \in \mathfrak{A}^{*}$ by $\langle\Gamma, F\rangle=\left\langle\Gamma_{1}, F^{\sharp}\right\rangle$. As $\Gamma=\Gamma_{1}=\gamma$ on $\mathfrak{A}_{0}$, and $\Gamma$ is ${ }^{+} \alpha$-invariant, we are done.

By V.7, $\mathfrak{A}_{0}$ can be identified with the disk algebra, and so if $G \in \mathfrak{A}_{0}$ we will let $G(\lambda)$ denote the value of the analytic function corresponding to $G$ at the point $\lambda$ in the closed unit disk. By the Lemma, there is a unique ${ }^{+} \alpha$-invariant linear functional $\Gamma^{(\lambda)} \in \mathfrak{A}^{*}$ such that

$$
\left\langle\Gamma^{(\lambda)}, F\right\rangle=F^{\sharp}(\lambda), \quad 0 \leq|\lambda| \leq 1,
$$

$F \in \mathfrak{A}$. Let $\Gamma_{x}^{(\lambda)}$ be as in V.2, V.3, and $R^{(\lambda)}=\left\{\Gamma_{x}^{(\lambda)}: x \in X\right\}_{\perp}$. On the dense subalgebra $K\left(\mathbf{Z}^{+}, C_{0}(X)\right) \subset \mathfrak{A}_{0},\left\langle\Gamma_{x}^{(\lambda)}, F\right\rangle=F^{\sharp}(\lambda), x \in X$. As observed in V.2, $\mathcal{R}^{(\lambda) \perp}=\left\{\Gamma \in \mathfrak{A}^{*}: \Gamma=\int_{X} \Gamma_{x}^{(\lambda)} d \mu(x)\right.$ for some $\mu \in \mathcal{M}(X)$; since $\mathcal{M}(X)$ contains a unique invariant probability measure $\omega$ and $\int_{X}\left\langle\Gamma_{x}^{(\lambda)}, F\right\rangle d \omega(x)=F^{\sharp}(\lambda)$, it follows that $\Gamma^{(\lambda)}=\int_{X} \Gamma_{x}^{(\lambda)} d \omega(x)$. Observe that the smallest closed $C(X)$-submodule $S$ of $\mathfrak{A}^{*}$ which contains $\Gamma^{(\lambda)}$ is exactly $R^{(\lambda) \perp}$ : let $\left\{f_{i}\right\}_{i=1}^{\infty} \subset C(X)$ satisfy $f_{i} \geq 0$, $\int f_{i} d \omega=1$, all $i$, and $\operatorname{supp}\left(f_{i}\right)$ runs through a neighborhood base at a given $x_{0} \in X$. Since the map $x \mapsto \Gamma_{x}^{(\lambda)}$ is continuous from $X$ to $\mathfrak{A}^{*}$ in the $\sigma\left(\mathfrak{A}^{*}, \mathfrak{A}\right)$ topology, $f_{i} \Gamma^{(\lambda)} \rightarrow \Gamma_{x_{0}}^{(\lambda)}$. Thus $\Gamma_{x_{0}}^{(\lambda)} \in S$ for each $x_{0} \in X$, and so $S=R^{(\lambda) \perp}$.

THEOREM. Every maximal $\alpha$-invariant right ideal of $\mathfrak{A}$ is of the form $R^{(\lambda)}$ for some complex number $\lambda,|\lambda| \leq 1$. If $\lambda \neq 0$, the ideal $\mathcal{R}^{(\lambda)}$ is maximal among all right ideals of $\mathfrak{A}$.

PROOF. The second statement is Corollary V.4. Let $R$ be a maximal $\alpha$-invariant right ideal. By V.8, $R^{\sharp}$ is a maximal ideal in the disk algebra, so $R^{\sharp}$ is the kernel of the evaluation map at some $\lambda$ in the closed unit disk [9, p. 87, Corollary]. As the functional $\gamma_{\lambda}: G \in \mathfrak{A}_{0} \rightarrow G(\lambda)$ belongs to $R^{\sharp \perp}$, it follows that $\Gamma^{(\lambda)} \subset R^{\perp}$. Since $R^{(\lambda) \perp}$ is the smallest closed $C(X)$-submodule of $\mathfrak{A}^{*}$ containing $\Gamma^{(\lambda)}$, and $R^{(\lambda) \perp}$ is ${ }^{+} \alpha$-invariant, the minimality $R^{\perp}$ forces $R^{\perp}=R^{(\lambda) \perp}$, or $R=R^{(\lambda)}$.

It remains only to show that $R^{(0)}$ is not maximal among all right ideals (although it is a maximal element in the collection of all $\alpha$-invariant right ideals). Let $F \in \mathfrak{A}$, $F=\sum_{n=0}^{\infty} U^{n} f_{n} ;$ now $\left\langle\Gamma_{x}^{(0)}, F\right\rangle=f_{0}(x)$, and $F \in R^{(0)}$ iff $F \in \operatorname{ker} \Gamma_{x}^{(0)}$ for all $x \in X$, iff $f_{0}=0$. Thus $R^{(0)}=U \mathfrak{A}$. Since, for each $x \in X$, $\operatorname{ker} \Gamma_{x}^{(0)}$ is a maximal two sided ideal (of codimension one) which properly contains $R^{(0)}, R^{(0)}$ is not a maximal right ideal.

V.11. Suppose $\mathfrak{A}$ is a Banach algebra with endomorphism $\alpha$; let us say that $\alpha$ acts on a right $\mathfrak{A}$-module $M$ if $M$ is isomorphic with $\mathfrak{A} / R$, where $R$ is an $\alpha$-invariant right ideal.

Suppose $(X, \phi)$ is a dynamical system and $Z \subset X$ is a compact minimal invariant subset. If $\phi^{-1}(Z)=Z$ and $J \sim(Z)$, then by Corollary IV.1, $\mathbf{Z}^{+} \times_{\phi} C_{0}(X) / J$ is isomorphic with $\mathbf{Z}^{+} \times_{\phi} C(Z)$, so there is a bijective correspondence between 
the maximal invariant right ideals of $\mathbf{Z}^{+} \times_{\phi} C_{0}(X)$ which contain $\mathrm{J}$ and those of $\mathbf{Z}^{+} \times_{\phi} C(Z)$. If, in addition, $(X, \phi)$ is free and $(Z, \phi)$ is uniquely ergodic, Theorem V.9 gives a complete classification of the irreducible right $\mathrm{Z}^{+} \times_{\phi} C_{0}(X)$-modules on which $\alpha$ acts, with kernel $J$. Unfortunately, these methods do not yield a classification in the case of an arbitrary right primitive ideal $\mathrm{J}$.

\section{REFERENCES}

1. W. Arveson, Ten lectures on operator algebras, CBMS Regional Conf. Ser. in Math., no. 55, Amer. Math. Soc., Providence, R. I., 1984.

2. W. Arveson and K. Josephson, Operator algebras and measure preserving automorphisms. II, J. Funct. Anal. 4 (1969), 100-134.

3. J. Brown, Ergodic theory and topological dynamics, Academic Press, New York, 1976.

4. W. Eberlein, Abstract ergodic theorems and weak almost periodic functions, Trans. Amer. Math. Soc. 67 (1949), 217-240.

5. E. Effros and F. Hahn, Locally compact transformation groups and $C^{*}$-algebras, Mem. Amer. Math. Soc. No. 75 (1967).

6. H. Furstenberg, Recurrence in ergodic theory and combinatorial number theory, Princeton Univ. Press, Princeton, N.J., 1981.

7. W. Gottschalk and G. Hedlund, Topological dynamics, Amer. Math. Soc. Colloq. Publ., no. 36, Amer. Math. Soc., Providence, R. I., 1955.

8. I. Herstein, Noncommutative rings, Carus Math. Monographs, No. 15, Wiley, New York, 1968.

9. K. Hoffman, Banach spaces of analytic functions, Prentice-Hall, Englewood Cliffs, N.J., 1962.

10. P. Muhly, Radicals, crossed products, and flows, Ann. Polon. Math. 43 (1983), 35-42.

11. J. Peters, Semi-crossed products of $C^{*}$-algebras, J. Funct. Anal. 599 (1984), 498-534.

12. C. Rickart, Banach algebras, Van Nostrand, Princeton, N. J., 1960.

13. B. Solel, Irreducible triangular algebras, Mem. Amer. Math. Soc. No. 47 (1984).

14. P. Walters, Ergodic theory-Introductory lectures, Lecture Notes in Math., vol. 458, SpringerVerlag, Berlin and New York, 1975.

Department of Mathematics, IOWA State University, Ames, IOWA 50011 